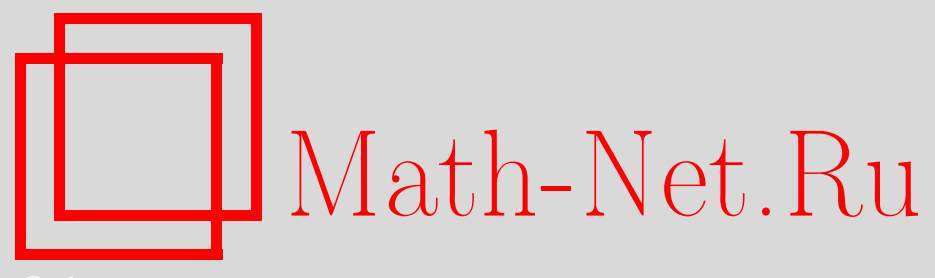

С. В. Полин, Заведомо разрешимые уравнения на алгебраических системах, Матем. вопр. криптогр., 2012, том 3, выпуск 4, 91-125

DOI: https://doi.org/10.4213/mvk69

Использование Общероссийского математического портала Math-Net.Ru подразумевает, что вы прочитали и согласны с пользовательским соглашением http: //www.mathnet.ru/rus/agreement

Параметры загрузки:

IP : 54.172 .240 .79

26 апреля 2023 г., 16:05:20 


\title{
Заведомо разрешимые уравнения на алгебраических системах
}

\author{
С. В. Полин \\ Академия криптографии Российской Федерации, Москва
}

Получено 20.V.2011

Исследуется возможность применения методов последовательного группирования для решения заведомо разрешимых уравнений.

Ключевые слова: заведомо разрешимые уравнения, заведомо совместные системы уравнений, метод группирования

\section{Certainly solvable equations on algebraic systems}

\section{S. V. Polin}

Academy of Cryptography of the Russian Federation, Moscow

\begin{abstract}
The applicability of successive grouping methods for solution of certainly solvable equations is studied.
\end{abstract}

Key words: certainly solvable equations, certainly compatible systems of equations, grouping method

Citation: Mathematical Aspects of Cryptography, 2012, vol. 3, no. 4, pp. 91-125 (Russian). 


\section{Введение}

Настоящая работа тесно примыкает к работе [6], в которой для нелинейных уравнений и систем нелинейных уравнений была рассмотрена общая модель класса алгоритмов их решения, состоящих из нескольких последовательных этапов, на каждом из которых проводится группирование неизвестных с последующим опробованием и отсевом группы. Также был предложен метод, позволяющий ускорить построение оптимального алгоритма из рассматриваемого класса для случайных уравнений с независимой правой частью.

Уравнения и системы уравнений с независимой правой частью обычно используются в качестве модели, описывающей случай ложного опробования части неизвестных. Случай истинного опробования описывается с помощью введенного Г. В. Балакиным [1] понятия заведомо совместных систем уравнений. В общем случае исследование заведомо совместных систем уравнений связано с некоторыми техническими трудностями. Чтобы преодолеть их, Г. В. Балакин ввел понятие заведомо совместных систем уравнений, согласованных с системами уравнений с независимыми правыми частями.

Одной из целей настоящей работы является обобщение понятия согласованности. Мы докажем, что для любого случайного уравнения $\Phi$ существует согласованное с ним заведомо разрешимое уравнение $\Psi$. Используя этот факт, мы для некоторых классов заведомо разрешимых уравнений рассчитаем параметры, необходимые для построения оптимального алгоритма последовательного опробования, и оценим его трудоемкость. Также будет оценена трудоемкость алгоритма, оптимизированного для случайного уравнения $\Phi$, в случае его применения при решении заведомо разрешимого уравнения $\Psi$.

\section{1. Конгруэнц-множества}

Пусть $A, B$ - произвольное множество. Введем обозначения.

- $B^{A}$ - множество всех отображений из множества $A$ в множество $B$.

- $\mathrm{E}(A)$ - решетка всех отношений эквивалентности на множестве $A$. Операции объединения и пересечения в этой решетке будем обозначать через $\vee$ и $\wedge$ соответственно ${ }^{1}$.

\footnotetext{
${ }^{1}$ Отметим, что операция $\wedge$ совпадает с теоретико-множественным пересечением, а операция $\vee$ в общем случае отличается от теоретико-множественного объединения.
} 
- $\Delta_{A}$ - отношение эквивалентности на множестве $A$, для которого $\left(a_{1}, a_{2}\right) \in \Delta_{A}$ тогда и только тогда, когда $a_{1}=a_{2}$.

- $\nabla_{A}$ - отношение эквивалентности на множестве $A$, для которого $\left(a_{1}, a_{2}\right) \in \nabla_{A}$ при всех $\left(a_{1}, a_{2}\right) \in A$.

Элементы $\Delta_{A}, \nabla_{A}$ являются наименьшим и наибольшим элементами решетки $\mathcal{E}(A)$ соответственно.

В работе [6] уравнением называется каждая пара $(f, b)$, где $f$ является отображением $f: A \rightarrow B$ конечных множеств, $b \in B$. Обычно оно записывается в виде

$$
f(x)=b,
$$

где $x$ - некоторый терм.

Рассматриваемому отображению $f$ соответствует двухосновная алгебра $\mathfrak{A}_{f}=\langle A, B ; f\rangle$. В соответствии с общим определением [3] пара отношений $(\theta, \alpha) \in \mathcal{E}(A) \times \mathcal{E}(B)$, определенных на множествах $A, B$ соответственно, называется конгруэнщией алгебры $\mathfrak{A}_{f}$, если справедливо высказывание

$$
\forall a, a^{\prime} \in A:\left(\left(a, a^{\prime}\right) \in \theta\right) \Rightarrow\left(\left(f(a), f\left(a^{\prime}\right)\right) \in \alpha\right) .
$$

Допуская некоторую неточность, будем называть конгруэнции алгебры $\mathfrak{A}_{f}$ конгруэнщияли уравнения (1.1).

Пусть пара $(\theta, \alpha)$ является конгруэнцией уравнения (1.1), $\pi_{\theta}: A \rightarrow A / \theta, \sigma_{\alpha}: B \rightarrow B / \alpha$ - естественные гомоморфизмы. Тогда существует единственное отображение $f_{\theta \alpha}: A / \theta \rightarrow B / \alpha$, удовлетворяющее условию $f_{\theta \alpha} \pi_{\theta}=\sigma_{\alpha} f$. Это позволяет рассмотреть уравнение

$$
f_{\theta \alpha}(y)=\sigma_{\alpha}(b) .
$$

Исходя из стандартной алгебраической терминологии, его следует называть фактор-уравнением. Ясно, что если элемент $a \in A$ является решением уравнения (1.1), то элемент $\pi_{\theta}(a)$ является решением уравнения (1.3). На этом свойстве основан метод гомоморфизма, позволяющий в определенных случаях решать уравнение (1.1) с трудоемкостью, меньшей трудоемкости тотального перебора [3]. 
К сожалению, решетки $\mathcal{E}(A)$ и $\mathcal{E}(B)$ устроены довольно сложно. Поэтому поиск конгруэнций и исследование построенных с их помощью алгоритмов связаны с серьезными техническими трудностями. В связи с этим приходится накладывать на используемые конгруэнции дополнительные ограничения.

Выбор ограничений определяется практической задачей. Так, типичным является случай, когда множества $A$ и $B$ являются носителями некоторых алгебраических систем А и В соответственно. Например, эти системы могут быть векторными пространствами, группами или кольцами. В этом случае можно рассматривать только такие конгруэнции $(\theta, \alpha)$, что отношения $\theta$ и $\alpha$ являются конгруэнциями систем $\mathbf{A}$ и В. Хотя именно этот случай представляет для нас наибольший интерес, мы пойдем другим путем, позволяющим провести рассмотрения в наибольшей общности.

Напомним [4, с. 252], что представлением решетки $\mathcal{R}$ называется произвольный мономорфизм $\eta: \mathcal{R} \rightarrow \mathcal{E}(C)$ из решетки $\mathcal{R}$ в решетку отношений эквивалентности какого-либо множества. Мы будем рассматривать только представления, удовлетворяющие дополнительному условию $\eta\left(1_{\mathcal{R}}\right)=\nabla_{C}$, $\eta\left(0_{\mathcal{R}}\right)=\Delta_{C}$, где $1_{\mathcal{R}}, 0_{\mathcal{R}}$ - наибольший и наименьший элементы решетки $\mathcal{R}$.

Начиная с данного момента, будем считать, что заданы конечные решетки $\mathcal{L}, \mathcal{M}$ и их представления $\xi: \mathcal{L} \rightarrow \mathcal{E}(A), \zeta: \mathcal{M} \rightarrow \mathcal{E}(B)$. (Позднее на решетки и представления будут наложены дополнительные ограничения.) Конгруэнцию $(\theta, \alpha)$ алгебры $\mathfrak{A}_{f}$ или, что то же самое, уравнения (1.1), назовем допустимой, если $\theta \in \xi(\mathcal{L}), \alpha \in \zeta(\mathcal{M})$.

В связи со сделанными выше замечаниями можно ожидать, что хотя бы некоторые свойства уравнения (1.1) зависят от множества допустимых конгруэнций алгебры $\mathfrak{A}_{f}$. Опять же, следуя стандартной алгебраической терминологии, такие свойства будем называть конгруэни-свойствами. Именно такие свойства нас и будут интересовать. Нам потребуются некоторые обозначения и определения.

Для каждой пары $(\theta, \alpha) \in \mathcal{L} \times \mathcal{M}$ через $(\theta, \alpha)^{\perp}$ обозначим множество таких отображений $f \in B^{A}$, что пара $(\xi(\theta), \zeta(\alpha))$ является конгруэнцией алгебры $\mathfrak{A}_{f}$. Для произвольного подмножества $\Gamma \subseteq \mathcal{L} \times \mathcal{M}$ положим $\Gamma^{\perp}=\bigcap_{(\theta, \alpha) \in \Gamma}(\theta, \alpha)^{\perp}$. Каждое множество вида $\Gamma^{\perp}$ будем называть конгруэнцмножеством. 
Ясно, что исследование конгруэнц-свойств по сути эквивалентно исследованию конгруэнц-множеств. Поэтому возникает задача их описания.

Пусть $\mathcal{K}, \mathcal{R}$ - решетки с наибольшими элементами $1_{\mathcal{K}}$ и $1_{\mathcal{R}}$ соответственно. Тогда отображение $v: \mathcal{K} \rightarrow \mathcal{R}$ называется нижним гомоморфизмом, если $v\left(1_{\mathcal{K}}\right)=1_{\mathcal{R}}$ и $v(\alpha \wedge \beta)=v(\alpha) \wedge v(\beta)$ при всех $\alpha, \beta \in \mathcal{K}$. Двойственным образом определяется понятие верхнего гомоморфизма.

Приведем некоторые свойства таких гомоморфизмов.

Лемма 1.1. Суперпозиция нижних (верхних) гомоморфизмов является нижним (верхним) гомоморфизмом.

Утверждение является частным случаем хорошо известного утверждения о суперпозиции гомоморфизмов универсальных алгебр.

Будем говорить, что нижний гомоморфизм $v: \mathcal{M} \rightarrow \mathcal{L}$ и верхний гомоморфизм $\tau: \mathcal{L} \rightarrow \mathcal{M}$ сопряжены, если справедливо высказывание

$$
\forall \alpha \in \mathcal{M}, a \in \mathcal{L}:(v(\alpha) \geq a) \Leftrightarrow(\alpha \geq \tau(a)) .
$$

Лемма 1.2. Для любого нижнего гомоморфизма $v: \mathcal{K} \rightarrow \mathcal{R}$ конечных решеток найдется единственный сопряженный ему верхний гомоморфизм $\tau: \mathcal{R} \rightarrow \mathcal{K}$.

Доказательство содержится в работе [6].

Следствие 1.1. Существует такой нижний гомоморфизм $\rho: \mathcal{E}(A) \rightarrow \mathcal{L}$, что справедливо высказывание

$$
\forall a \in \mathcal{E}(A), \alpha \in \mathcal{L}:(\xi(\alpha) \leq a) \Leftrightarrow(\alpha \leq \rho(a)) .
$$

Нетрудно видеть, что отображение $\xi: \mathcal{L} \rightarrow \mathcal{E}(A)$ является верхним гомоморфизмом. Из утверждения, двойственного лемме 1.2 , вытекает существование такого нижнего гомоморфизма $\rho: \mathcal{E}(A) \rightarrow \mathcal{L}$, что гомоморфизмы $\rho, \xi$ сопряжены. Высказывание (1.5) как раз и выражает их сопряженность.

Теорема 1.1. Для любого отображения $f: A \rightarrow B$ существуют такой нижний гомоморфизм $v: \mathcal{M} \rightarrow \mathcal{L}$ u такой верхний гомоморфизм $\tau: \mathcal{L} \rightarrow \mathcal{M}$, что множество $\mathcal{C}\left(\mathfrak{A}_{f}\right)$ допустимых конгруэнций алгебры $\mathfrak{A}_{f}$ совпадает с каждым из множеств

$$
\begin{aligned}
\mathfrak{H}(v) & =\langle(\xi(\theta), \zeta(\alpha)) \in \xi(\mathcal{L}) \times \zeta(\mathcal{M}) \mid \theta \leq v(\alpha)\rangle, \\
\mathfrak{G}(\tau) & =\langle(\xi(\theta), \zeta(\alpha)) \in \xi(\mathcal{L}) \times \zeta(\mathcal{M}) \mid \tau(\theta) \leq \alpha\rangle .
\end{aligned}
$$


Пусть $\alpha \in \mathcal{M}$. Полагаем

$$
\omega(\alpha)=\left\langle\left(a_{1}, a_{2}\right) \in A \mid\left(f\left(a_{1}\right), f\left(a_{2}\right)\right) \in \zeta(\alpha)\right\rangle .
$$

Тем самым построено отображение $\omega: \mathcal{M} \rightarrow \mathcal{E}(A)$.

По определению представлений $\zeta\left(1_{\mathcal{M}}\right)=\nabla_{B}$. Отсюда легко следует, что $\omega\left(1_{\mathcal{M}}\right)=\nabla_{A}$.

Как мы уже отмечали, пересечение в решетке $\mathcal{E}(A)$ совпадает с теоретико-множественным пересечением. Поэтому имеем

$$
\begin{gathered}
\left(\left(a_{1}, a_{2}\right) \in \omega(\alpha \wedge \beta)\right) \Leftrightarrow\left(\left(f\left(a_{1}\right), f\left(a_{2}\right)\right) \in \zeta(\alpha \wedge \beta)\right) \Leftrightarrow \\
\Leftrightarrow\left(\left(f\left(a_{1}\right), f\left(a_{2}\right)\right) \in \zeta(\alpha) \wedge \zeta(\beta)\right) \Leftrightarrow \\
\Leftrightarrow\left(\left(\left(f\left(a_{1}\right), f\left(a_{2}\right)\right) \in \zeta(\alpha)\right) \&\left(\left(f\left(a_{1}\right), f\left(a_{2}\right)\right) \in \zeta(\beta)\right)\right) \Leftrightarrow \\
\Leftrightarrow\left(\left(a_{1}, a_{2}\right) \in \omega(\alpha) \wedge \omega(\beta)\right) .
\end{gathered}
$$

Таким образом, $\omega(\alpha \wedge \beta)=\omega(\alpha) \wedge \omega(\beta)$ и отображение $\omega$ является нижним гомоморфизмом решеток.

По следствию 1.1 существует нижний гомоморфизм $\rho: \mathcal{E}(A) \rightarrow \mathcal{L}$, для которого справедливо высказывание (1.5). Суперпозицию $\rho \omega: \mathcal{M} \rightarrow \mathcal{L}$ обозначим через $v$. В силу леммы 1.1 отображение $v$ является нижним гомоморфизмом.

Пусть $\theta \in \mathcal{L}, \alpha \in \mathcal{M}$. По определению пара $(\xi(\theta), \zeta(\alpha))$ является конгруэнцией алгебры $\mathfrak{A}_{f}$ тогда и только тогда, когда

$$
\forall a_{1}, a_{2} \in A:\left(\left(a_{1}, a_{2}\right) \in \xi(\theta)\right) \Rightarrow\left(\left(f\left(a_{1}\right), f\left(a_{2}\right)\right) \in \zeta(\alpha)\right) .
$$

Легко видеть, что это высказывание эквивалентно неравенству $\omega(\alpha) \geq \xi(\theta)$. Из формулы (1.5) следует, что последнее неравенство эквивалентно неравенству $v(\alpha)=\rho(\omega(\alpha)) \geq \theta$. Это неравенство выполняется тогда и только тогда, когда $(\xi(\theta), \zeta(\alpha)) \in \mathfrak{H}(v)$, т. е. множество $\mathfrak{H}(v)$ совпадает с множеством допустимых конгруэнций алгебры $\mathfrak{A}_{f}$.

Наконец, в качестве $\tau$ возьмем верхний гомоморфизм, сопряженный нижнему гомоморфизму $v$. Из сопряженности гомоморфизмов $v, \tau$ вытекает равенство $\mathfrak{H}(v)=\mathfrak{G}(\tau)$. 
Теорема 1.2. Пусть $v: \mathcal{M} \rightarrow \mathcal{L}-$ произвольный нижний гомоморфизм. Тогда следующие утверждения эквивалентны.

1. Каждая пара из множества $\mathfrak{H}(v)$ является допустимой конгруэнщией алгебры $\mathfrak{A}_{f}$.

2. Для каждого элемента $\alpha \in \mathcal{M}$ пара $(\xi v(\alpha), \zeta(\alpha))$ является допустимой конгруэнцией алгебры $\mathfrak{A}_{f}$.

3. Для каждого элемента $\alpha \in \mathcal{M}$ имеет место включение

$$
\operatorname{ker}\left(\sigma_{\alpha} f\right) \supseteq \xi v(\alpha),
$$

где $\sigma_{\zeta(\alpha)}: B \rightarrow B / \zeta(\alpha)$ - естественный эпиморфизм.

Пусть справедливо утверждение 1.

Заметим, что $v(\alpha) \leq v(\alpha)$. Отсюда и из определения множества $\mathfrak{H}(v)$ следует, что каждая пара $(\xi v(\alpha), \zeta(\alpha))$ принадлежит множеству $\mathfrak{H}(v)$. Отсюда очевидным образом вытекает импликация $1 \Rightarrow 2$.

По определению пара $(\xi v(\alpha), \zeta(\alpha))$ является допустимой конгруэнцией алгебры $\mathfrak{A}_{f}$ тогда и только тогда, когда справедливо высказывание

$$
\forall a, a^{\prime} \in A:\left(\left(a, a^{\prime}\right) \in \xi v(\alpha)\right) \Rightarrow\left(\left(f(a), f\left(a^{\prime}\right)\right) \in \zeta(\alpha)\right) .
$$

Нетрудно видеть, что высказывание (1.7) является развернутой записью высказывания (1.6). Поэтому эти высказывания эквивалентны и $2 \Leftrightarrow 3$.

Пусть справедливо утверждение 2. Из предыдущего абзаца следует, что при сделанном предположении справедливо высказывание (1.7). Если $(\xi(\theta), \zeta(\alpha)) \in \mathfrak{H}(v)$, то $\theta \leq v(\alpha)$. Так как каждый гомоморфизм решеток является монотонным отображением, то $\xi(\theta) \leq \xi v(\alpha)$, т. е. справедливо высказывание

$$
\forall a, a^{\prime} \in A:\left(\left(a, a^{\prime}\right) \in \xi(\theta)\right) \Rightarrow\left(\left(a, a^{\prime}\right) \in \xi v(\alpha)\right) .
$$

Объединив его с высказыванием (1.7), получаем

$$
\forall a, a^{\prime} \in A:\left(\left(a, a^{\prime}\right) \in \xi(\theta)\right) \Rightarrow\left(\left(f(a), f\left(a^{\prime}\right)\right) \in \zeta(\alpha)\right) .
$$

Таким образом, пара $(\xi(\theta), \zeta(\alpha))$ является допустимой конгруэнцией алгебры $\mathfrak{A}_{f}$ и $2 \Rightarrow 1$. 
Через $\mathfrak{F}(A, B ; v)$ обозначим множество всех отображений $f: A \rightarrow B$, для которых выполняются все включения вида (1.6). Из доказанного следует, что каждое такое множество является конгруэнц-множеством. Более того, нетрудно видеть, что каждое конгруэнц-множество является пересечением некоторого числа множеств вида $\mathfrak{F}(A, B ; v)$.

Таким образом, приходим к необходимости исследовать в первую очередь именно множества $\mathfrak{F}(A, B ; v)$.

\section{2. Случайные и заведомо разрешимые случайные уравнения}

В соответствии с общим определением каждый элемент множества $\mathbb{U}(A, B ; v)=\mathfrak{F}(A, B ; v) \times B$ является уравнением. Для каждого уравнения $U \in \mathbb{U}(A, B ; v)$ через $\operatorname{Sol}(U)$ обозначим множество его решений. Полагаем

$$
\mu_{a}(U)=\left\{\begin{array}{ll}
1, & a \in \operatorname{Sol}(U) ; \\
0, & a \notin \operatorname{Sol}(U) ;
\end{array} \quad \eta(U)=\sum_{a \in A} \mu_{a}(U) .\right.
$$

Таким образом, $\mu_{a}(U)$ есть индикатор события «элемент $a$ является решением уравнения $U \gg$, а $\eta(U)$ - число решений уравнения $U$.

Если $\operatorname{Sol}(U) \neq \varnothing$, то уравнение $U$ будем называть разрешимылм. Множество всех разрешимых уравнений обозначим через $\mathbb{U}_{R}(A, B ; v)$. Другими словами,

$$
\mathbb{U}_{R}(A, B ; v)=\langle U \in \mathbb{U}(A, B ; v) \mid \eta(U)>0\rangle .
$$

Если $U=(f, b)$, то вместо $\operatorname{Sol}((f, b))$ будем писать $\operatorname{Sol}(f, b)$. То же самое касается и других функций, определенных на множестве $\mathbb{U}(A, B ; v)$.

Напомним определение [8, с. 221].

Пусть $\langle Y ; \mathfrak{B}, \mathrm{P}\rangle$ - вероятностное пространство, $\langle E ; \mathfrak{E}\rangle$ - измеримое пространство ${ }^{1}$. Тогда будем говорить, что функция $\varphi: Y \rightarrow E$ является случайным элементом со значениями в $E$ или короче случайныл Е-элементом, если эта функция является измеримым отображением пространств $\varphi:\langle Y ; \mathfrak{B}\rangle \rightarrow\langle E ; \mathfrak{E}\rangle$.

\footnotetext{
${ }^{1}$ Отметим, что в ряде работ, например, в монографии [10, с. 28] измеримые пространства называются $\sigma$-алгебрами. Мы выбрали первый термин как более распространенный в теоретико-вероятностной литературе.
} 
Последнее означает, что для каждого подмножества $B \in \mathfrak{E}$ его прообраз $\varphi^{-1}(B)=\langle y \in Y \mid \varphi(y) \in B\rangle$ принадлежит множеству $\mathfrak{B}$.

Если отображения $\varphi:\langle Y ; \mathfrak{B}, \mathrm{P}\rangle \rightarrow\langle E ; \mathfrak{E}\rangle$ и $\psi:\langle E ; \mathfrak{E}\rangle \rightarrow\langle D ; \mathfrak{D}\rangle$ измеримы, то суперпозиция $\psi \varphi:\langle Y ; \mathfrak{B}, \mathrm{P}\rangle \rightarrow\langle D ; \mathfrak{D}\rangle$ также измерима. Поэтому эта суперпозиция является случайным элементом со значениями в $D$. Будем обозначать этот случайный элемент $\psi(\varphi)$.

Пусть $X$ - произвольное конечное множество. Тогда его можно рассматривать как дискретное измеримое пространство $\left\langle X, 2^{X}\right\rangle$, в котором любое подмножество измеримо. Это позволяет определить случайные $X$-элементы, как такие отображения $\gamma: Y \rightarrow X$ из носителя $Y$ вероятностного пространства $\langle Y ; \mathfrak{B}, \mathrm{P}\rangle$ в множество $X$, что прообраз $\gamma^{-1}(x)$ любой точки $x \in \mathbf{X}$ принадлежит множеству $\mathfrak{B}$.

Каждый случайный $X$-элемент $\gamma:\langle Y ; \mathfrak{B}, \mathrm{P}\rangle \rightarrow X$ индуцирует на множестве $X$ распределение вероятностей ${ }^{1} p_{\gamma}$, для которого

$$
p_{\gamma}(x)=P\left(\gamma^{-1}(x)\right) \text {. }
$$

Нетрудно видеть, что каждое отображение $\delta: X \rightarrow Z$ из множества $X$ в носитель измеримого пространства $\langle D ; \mathfrak{D}\rangle$ является измеримым отображением $\delta:\left\langle X, 2^{X}\right\rangle \rightarrow\langle D ; \mathfrak{D}\rangle$ пространств. Поэтому для каждого такого отображения $\delta$ определен случайный элемент $\delta(\gamma)$ со значениями в множестве $D$.

Поскольку рассматриваемое нами множество уравнений $\mathbb{U}(A, B ; v)$ конечно, к нему применимы вышеуказанные понятия. Это дает право говорить о случайных уравнениях со значениями в множестве $\mathbb{U}(A, B ; v)$. При этом проекции, отображающие множество $\mathbb{U}(A, B ; v)=\mathfrak{F}(A, B ; v) \times B$ на прямые сомножители, связывают с каждым случайным уравнением $\Phi$ случайную функцию $\operatorname{pr}_{\mathfrak{F}(A, B ; v)}(\Phi)$ (левую часть уравнения) и случайный $B$-элемент $\operatorname{pr}_{B}(\Phi)$ (правая часть).

\footnotetext{
${ }^{1}$ Распределением вероятностей на конечном или счётном множестве $X$ называется каждая неотрицательная функция $p$, заданная на множестве $X$ и удовлетворяющая условию $\sum_{x \in X} p(x)=1$ (см. [2, с. 14]).
} 
Пусть $\tau$ - отображение, удовлетворяющее равенству (1.4). Тогда для каждого элемента $\theta \in \mathcal{L}$ выполняется неравенство $\tau(\theta) \leq \tau(\theta)$. Поэтому паpa $(\xi(\theta), \zeta(\tau(\theta)))$ принадлежит множеству $\mathfrak{G}(\tau)$, определенному в теореме 1.1. В силу той же теоремы множество $\mathfrak{G}(\tau)$ совпадает с множеством $\mathfrak{H}(v)$. Отсюда и из теоремы 1.2 следует, что пара $(\xi(\theta), \zeta(\tau(\theta)))$ является конгруэнцией алгебры $\mathfrak{A}_{f}$ для каждого отображения $f \in \mathfrak{F}(A, B ; v)$ и, следовательно, конгруэнцией каждого уравнения $(f, b) \in \mathbb{U}(A, B ; v)$, что дает возможность определить уравнение $f_{\theta \tau(\theta)}(y)=\sigma_{\tau(\theta)}(b)$, которое мы будем обозначать $(f, b)^{\theta}$.

Полагаем $\mathcal{L}_{\theta}=\langle\rho \in \mathcal{L} \mid \rho \geq \theta\rangle$. В силу теорем об изоморфизме для каждого элемента $\rho \in \mathcal{L}_{\theta}$ на множестве $A / \xi(\theta)$ определено отношение эквивалентности $\xi_{\theta}(\rho)=\xi(\rho) / \xi(\theta)$. Легко проверяется, что отображение $\xi_{\theta}: \mathcal{L}_{\theta} \rightarrow \mathcal{E}(A / \xi(A))$ является представлением решетки $\mathcal{L}_{\theta}$. Точно так же определяем представление $\zeta_{\tau(\theta)}$ решетки $\mathcal{M}_{\tau(\theta)}=\langle\alpha \in \mathcal{M} \mid \alpha \geq \tau(\theta)\rangle$ отношениями эквивалентности на множестве $B / \zeta(\tau(\theta))$.

Пусть $\alpha \in \mathcal{M}_{\tau(\theta)}$. Это означает, что $\alpha \geq \tau(\theta)$. Применив равенство (1.4), получаем, что $v(\alpha) \geq \theta$, т. е. $v(\alpha) \in \mathcal{L}_{\theta}$. Пусть $v^{\theta}: \mathcal{M}_{\tau(\theta)} \rightarrow \mathcal{L}_{\theta}$ есть ограничение отображения $v: \mathcal{M} \rightarrow \mathcal{L}$ на подрешетку $\mathcal{M}_{\tau(\theta)}$.

Легко проверить, что отображение $v^{\theta}$ является нижним гомоморфизмом решеток и что для всех уравнений $(f, b) \in \mathbb{U}(A, B ; v)$ фактор-уравнение $(f, b)^{\theta}$ принадлежит множеству $\mathbb{U}\left(A / \xi(\theta), B / \zeta(\tau(\theta)) ; v^{\theta}\right)$.

Теперь в соответствии с общим определением по каждому случайному уравнению $\Phi$ со значениями в множестве $\mathbb{U}(A, B ; v)$ можно построить случайное уравнение $\Phi^{\theta}$ со значениями в множестве $\mathbb{U}\left(A / \xi(\theta), B / \zeta(\tau(\theta)) ; v^{\theta}\right)$. 
Случайное уравнение $\Phi$ назовем заведомо разрешилым ${ }^{1}$, если множество $\mathbb{U}_{R}(A, B ; v)$ является носителем его распределения вероятностей. Другими словами, вероятность неразрешимости случайного уравнения $\Phi$ равна 0.

Проведенные исследования случайных систем уравнений показывают, что случайные заведомо совместные системы уравнений, представляющие практический интерес, устроены сложнее, чем случайные системы общего вида, что затрудняет их изучение. Г. В. Балакин [1] показал, что для некоторых классов заведомо совместных систем уравнений существуют согласованные с ними случайные системы общего вида и что изучение таких классов заведомо совместных систем может быть сведено к изучению согласованных систем. Нашей ближайшей целью является обобщение понятия согласованности на произвольные заведомо разрешимые случайные уравнения.

Пусть $\Phi$ - случайное уравнение, $\Psi$ - заведомо разрешимое случайное уравнение. Будем говорить, что случайное уравнение $\Psi$ согласовано со случайным уравнением $\Phi$, если выполняются равенства

$$
\begin{aligned}
& \mathrm{E}(\eta(\Phi))^{k}=(\mathrm{E} \eta(\Phi)) \mathrm{E}(\eta(\Psi))^{k-1}, \quad k \geq 1, \\
& \mathrm{P}(\eta(\Phi)=0)=1-\mathrm{E} \eta(\Phi) \sum_{k \geq 1} \frac{\mathrm{P}(\eta(\Psi)=k)}{k}, \\
& \mathrm{P}(\eta(\Phi)=k)=\mathrm{E} \eta(\Phi) \frac{\mathrm{P}(\eta(\Psi)=k)}{k}, \quad k \geq 1 .
\end{aligned}
$$

Обоснованием такого определения служит его сравнение с теоремой 4.1 работы [1].

Теорема 2.1. Для любого случайного уравнения $\Phi$ найдется согласованное с ним заведомо разрешимое случайное уравнение $\Phi^{*}$ с истинным решением.

Доказательство. Если случайное уравнение $\Phi$ принимает значения только в дополнении подмножества $\mathbb{U}_{R}(A, B ; v)$, то из определения и равенства (2.2) следует, что любое заведомо разрешимое случайное уравнение $\Psi$ согласовано со случайным уравнением $\Phi$.

\footnotetext{
${ }^{1}$ По сути, наше определение совпадает с введенным Г. В. Балакиным понятием заведомо совместной системы уравнений. К сожалению, мы вынуждены были изменить устоявшийся термин, поскольку говорить о совместности одного уравнения было бы странно.
} 
Поэтому нам нужно рассмотреть только случай, когда $\Phi$ с ненулевой вероятностью принимает значения, принадлежащие множеству $\mathbb{U}_{R}(A, B ; v)$. Из равенства (2.2) легко выводится, что в этом случае имеем

$$
\mathrm{E} \eta(\Phi)>0 \text {. }
$$

Распределение вероятностей случайной величины $\Phi$ обозначим через $p$. Пусть $a \in A$. Полагаем

$$
\mathbb{U}_{a}(A, B ; v)=\langle(f, f(a)) \mid f \in \mathfrak{F}(A, B ; v)\rangle .
$$

Другими словами, $\mathbb{U}_{a}(A, B ; v)$ есть подмножество, элементами которого являются все уравнения из множества $\mathbb{U}(A, B ; v)$, имеющие одним из решений элемент $a$. Полагаем

$$
r(a)=\sum_{(f, b) \in \mathbb{U} a(A, B ; v)} p(f, b) .
$$

Ясно, что эта величина есть вероятность того, что элемент $a \in A$ является решением случайного уравнения $\Phi$. Из определений следуют равенства

$$
\begin{gathered}
\mathrm{E} \mu_{a}(\Phi)=r(a), \\
\mathrm{E} \eta(\Phi)=\sum_{a \in A} \mathrm{E} \mu_{a}(\Phi)=\sum_{a \in A} r(a) .
\end{gathered}
$$

Неравенство (2.4) позволяет следующим образом определить на множестве $\mathbb{V}(A, B ; v)=A \times \mathfrak{F}(A, B ; v)$ неотрицательную функцию $q$ :

$$
q(a, f)=\frac{p(f, f(a))}{\mathrm{E} \eta(\Phi)}
$$

Имеем

$$
\sum_{(a, f)} q(a, f)=\frac{\sum_{(a, f)} p(f, f(a))}{\mathrm{E} \eta(\Phi)}=\frac{\sum_{a} \sum_{f} p(f, f(a))}{\mathrm{E} \eta(\Phi)}=\frac{\sum_{a} r(a)}{\mathrm{E} \eta(\Phi)}=\frac{\mathrm{E} \eta(\Phi)}{\mathrm{E} \eta(\Phi)}=1 .
$$

Таким образом, функция $q$ является распределением вероятностей на множестве $\mathbb{V}(A, B ; v)$. Она порождает вероятностную меру, для которой

$$
Q(W)=\sum_{V \in W} q(V)
$$

Теперь $\langle\mathbb{V}(A, B ; v) ; Q\rangle$ становится вероятностным пространством. 
Определим $\quad$ отображение $\Phi^{*}: \mathbb{V}(A, B ; v) \rightarrow \mathbb{U}(A, B ; v), \quad$ положив $\Phi^{*}(a, f)=(f, f(a))$. Тем самым построено случайное уравнение, распределение вероятностей которого определяется следующим образом:

$$
\begin{gathered}
p^{*}(f, b)=\sum_{a \in \operatorname{Sol}(f, b)} q(a, f)=\sum_{a \in \operatorname{Sol}(f, b)} \frac{p(f, f(a))}{\mathrm{E} \eta(\Phi)}= \\
=\sum_{a \in \operatorname{Sol}(f, b)} \frac{p(f, b)}{\operatorname{E} \eta(\Phi)}=\frac{\eta(f, b)}{\operatorname{E} \eta(\Phi)} p(f, b) .
\end{gathered}
$$

Рассмотрим произвольную функцию $h$, определенную на множестве $\mathbb{U}(A, B ; v)$. Имеем

$$
\begin{gathered}
\mathrm{E} h\left(\Phi^{*}\right)=\sum_{(f, b)} h(f, b) p^{*}(f, b)=\sum_{(f, b)} h(f, b) \frac{\eta(f, b)}{\mathrm{E} \eta(\Phi)} p(f, b)= \\
=\frac{1}{\mathrm{E} \eta(\Phi)} \sum_{(f, b)}(h(f, b) \eta(f, b)) p(f, b)=\frac{\mathrm{E}(h(\Phi) \eta(\Phi))}{\mathrm{E} \eta(\Phi)} .
\end{gathered}
$$

Взяв в качестве $h$ функцию $\eta^{k-1}$, получаем равенство (2.3).

Дословным повторением доказательства теоремы 4.1 работы [1] показываем, что оставшиеся равенства вытекают из доказанного соотношения между моментами.

Подчеркнем, что даже в случае, когда исходное уравнение $\Phi$ заведомо разрешимо, уравнения $\Phi$ и $\Phi^{*}$ могут иметь различные распределения. Более того, равенство (2.5) показывает, что для совпадения распределений необходимо и достаточно, чтобы все уравнения из носителя случайного уравнения $\Phi$ имели одинаковое число решений.

Может представлять интерес конструктивное описание процедуры выбора случайного уравнения $\Phi^{*}$.

1. Случайно выбираем элемент $a \in A$. Вероятность того, что выбрано значение $a$, равна $R(a)=\frac{r(a)}{\mathrm{E} \xi(\Phi)}$.

2. Случайно выбираем левую часть уравнения. Вероятность того, что выбрано значение $f$, равна $q_{a}(f)=\frac{p(f, f(a))}{r(a)}$.

3. Выбираем уравнение $(f, f(a))$. 
Доказанная теорема допускает обращение.

Теорема 2.2. Пусть $\Psi$ - случайное уравнение, принимающее с ненулевой вероятностью только значения из подмножества $\mathbb{U}_{R}(A, B ; v)$. Тогда существует такое случайное уравнение $\Phi$, что уравнения $\Psi$ и $\Phi^{*}$ имеют одинаковые распределения вероятностей.

Доказательство. Распределение вероятностей случайного уравнения $\Psi$ обозначим через $s$. Положим

$$
\begin{gathered}
E=\sum_{(f, b) \in \mathbb{U}_{R}(A, B ; v)} \frac{s(f, b)}{\eta(f, b)}, \\
p(f, b)=\left\{\begin{array}{cc}
\frac{s(f, b)}{E \eta(f, b)}, & \eta(f, b)>0 ; \\
0, & \eta(f, b)=0 .
\end{array}\right.
\end{gathered}
$$

Легко видеть, что построенная функция является распределением вероятностей на множестве $\mathbb{U}(A, B ; v)$. Этому распределению соответствует вероятностная мера $P$. Пусть $\Phi-$ случайное уравнение, распределение которого равно функции $p$, например, случайное уравнение

$$
\mathrm{id}_{\mathbb{U}(A, B ; v)}: \mathbb{U}(A, B ; v) \rightarrow \mathbb{U}(A, B ; v) .
$$

Из доказательства теоремы 2.1 следует, что распределением случайного уравнения $\Phi^{*}$ является функция

$$
p^{*}(f, b)=\frac{\eta(f, b)}{\mathrm{E} \eta(\Phi)} p(f, b) .
$$

Если $\eta(f, b)>0$, то

$$
p^{*}(f, b)=\frac{\eta(f, b)}{\mathrm{E} \eta(\Phi)} \frac{s(f, b)}{E \eta(f, b)}=\frac{s(f, b)}{E \mathrm{E} \eta(\Phi)} .
$$

Если $\eta(f, b)=0$, то из условий теоремы следует $s(f, b)=0$ и

$$
p *(f, b)=0=\frac{s(f, b)}{E \mathrm{E} \eta(\Phi)} .
$$


Далее. Из свойств вероятностных распределений следует

$$
1=\sum_{(f, b) \in \mathbb{U}(A, B ; v)} p^{*}(f, b)=\frac{1}{E \mathrm{E} \xi(\Phi)} \sum_{(f, b) \in \mathbb{U}(A, B ; v)} s(f, b)=\frac{1}{E \mathrm{E} \xi(\Phi)} .
$$

Таким образом, $E \mathrm{E} \xi(\Phi)=1$, и утверждение теоремы верно.

Таким образом, для каждого заведомо разрешимого случайного уравнения $\Psi$ найдется согласованное с ним случайное уравнение $\Phi$.

\section{3. Фактор-уравнения заведомо совместного уравнения}

Начиная с данного момента, будем считать, что рассматриваемые представления $\xi: \mathcal{L} \rightarrow \mathcal{E}(A), \zeta: \mathcal{M} \rightarrow \mathcal{E}(B)$ решеток перестановочны и однородны. Перестановочность означает, что отношения $\xi\left(\theta_{1}\right), \xi\left(\theta_{2}\right)$ $\left(\zeta\left(\beta_{1}\right), \zeta\left(\beta_{2}\right)\right)$ перестановочны [5] при всех $\theta_{1}, \theta_{2} \in \mathcal{L}\left(\beta_{1}, \beta_{2} \in \mathcal{M}\right)$, а однородность - что все классы эквивалентности каждого отношения $\xi(\theta)(\zeta(\alpha))$ имеют одинаковую мощность. Так как число классов отношения $\xi(\theta)$, где $\theta \in \mathcal{L}$, равно $|A / \xi(\theta)|$, то мощность каждого класса равна $|A||A / \xi(\theta)|^{-1}$. Аналогично, мощность каждого класса отношения $\zeta(\beta)$, где $\beta \in \mathcal{M}$, равна $|B||B / \zeta(\beta)|^{-1}$.

В работе [6] было показано, что в этих условиях с каждой максимальной цепью $\vec{\theta}$

$$
1_{\mathcal{L}}=\theta_{0}>\theta_{1}>\ldots>\theta_{m-1}=0_{\mathcal{L}}
$$

связан переборный алгоритм решения любого случайного уравнения $\Psi$, принимающего значения в множестве $\mathbb{U}(A, B ; v)$. Средняя трудоемкость алгоритма равна

$$
T(\Psi ; \vec{\theta})=\sum_{i=0}^{m-1}\left|A / \theta_{i+1}\right|\left|A / \theta_{i}\right|^{-1} \mathrm{E} \eta\left(\Psi^{\theta_{i}}\right)
$$

Так как множество цепей конечно, то на одной из них достигается минимальное значение трудоемкости. Это значение будем обозначать через $T(\Psi)$. 
Из формулы (3.1) ясно, что анализ и оптимизация таких алгоритмов требует вычисления величин $\mathrm{E} \xi\left(\Psi^{\theta}\right)$. Нашей ближайшей целью является их вычисление для заведомо разрешимого случайного уравнения $\Psi$, согласованного со случайным уравнением $\Phi$ с независимой, равномерно распределенной правой частью. Другими словами, пара $(f, b)$ является значением случайного уравнения $\Phi$ с вероятностью $\frac{1}{|B|} p(f)$, где $p$ - некоторое распределение, определенное на множестве $\mathfrak{F}(A, B ; v)$.

Свойство однородности можно уточнить. Для этого введем на множествах $A, B$ функции расстояния. На множестве $A$ расстояние определяется следующим образом (см. [4, с. 252]):

$$
\llbracket a_{1}, a_{2} \rrbracket=\min \left\langle\rho \in \mathcal{L} \mid\left(a_{1}, a_{2}\right) \in \xi(\rho)\right\rangle .
$$

Заметим, что для любого элемента $a \in A$ имеет место включение $(a, a) \in \Delta_{A}=\xi\left(0_{\mathcal{L}}\right)$. Поэтому $\llbracket a, a \rrbracket=0_{\mathcal{L}}$.

Расстояние на множестве $B$ определяется аналогично с использованием решетки $\mathcal{M}$.

В этих обозначениях класс $\xi(\theta)$-эквивалентности элемента $a \in A$ представляет собой шар $\mathcal{S}_{\theta}(a)=\left\langle a^{\prime} \in A \mid \llbracket a, a^{\prime} \rrbracket \leq \xi(\theta)\right\rangle$. Таким образом, записи $a^{\prime} \in \mathcal{S}_{\theta}(a), a \in \mathcal{S}_{\theta}\left(a^{\prime}\right)$ и $\left(a, a^{\prime}\right) \in \xi(\theta)$ эквивалентны. Наряду с шаром $\mathcal{S}_{\theta}(a)$ можно рассмотреть сферу $\mathcal{C}_{\theta}(a)=\left\langle a^{\prime} \in A \mid \llbracket a, a^{\prime} \rrbracket=\xi(\theta)\right\rangle$. Однородность решетки $\mathcal{L}$ означает, что мощности всех шаров заданного радиуса $\theta$ одинаковы. Покажем, что то же самое верно и для сфер.

Функцию Мёбиуса, определенную на решетке $\mathcal{L}$ [7, с. 72], обозначим через $\mu_{\mathbb{L}}$. Как известно, ее можно построить рекуррентным методом, используя следующие соотношения:

$$
\mu_{\mathcal{L}}(\theta, \theta)=1, \quad \mu_{\mathcal{L}}(\theta, \rho)=-\sum_{\langle\tau \mid \theta \leq \tau<\rho\rangle} \mu_{\mathcal{L}}(\theta, \tau) .
$$

Для любых элементов $a \in A, \theta \in \mathcal{L}$ имеем $\mathcal{S}_{\theta}(a)=\bigcup_{\langle\rho \in \mathcal{L} \mid \rho \leq \theta\rangle} \mathcal{C}_{\rho}\left(a_{1}\right)$, причем различные сферы, входящие в объединение, не пересекаются. Поэтому

$$
\sum_{\langle\rho \in \mathcal{L} \mid \rho \leq \theta\rangle}\left|\mathcal{C}_{\rho}(a)\right|=\left|\mathcal{S}_{\theta}(a)\right|=|A||A / \xi(\theta)|^{-1} .
$$


Теперь из свойств функции Мёбиуса следует

$$
\left|\mathcal{C}_{\theta}(a)\right|=\sum_{\langle\rho \in \mathcal{L} \mid \rho \leq \theta\rangle} \frac{|A|}{|A / \xi(\eta)|} \mu_{\mathcal{L}}(\eta, \theta) .
$$

Поскольку правая часть не зависит от элемента $a$, то все сферы радиуса $\theta$ имеют одинаковую мощность, которую мы будем обозначать $N_{\mathcal{L}}(\theta)$.

Точно так же мощность каждой сферы $\mathcal{C}_{\alpha}(b)=\left\langle b^{\prime} \in A \mid \llbracket b, b^{\prime} \rrbracket=\zeta(\alpha)\right\rangle$ зависит только от радиуса $\alpha$ и равна

$$
N_{\mathcal{M}}(\alpha)=\sum_{\langle\beta \in \mathcal{M} \mid \beta \leq \alpha\rangle} \frac{|B|}{|B / \zeta(\beta)|} \mu_{\mathcal{M}}(\beta, \alpha) .
$$

Пусть $\varphi$ - левая часть случайного уравнения. Для элементов $a_{1}, \ldots, a_{k} \in A, b_{1}, \ldots, b_{k} \in B$ полагаем

$$
\begin{gathered}
P\left(a_{1}, \ldots, a_{k} ; b_{1}, \ldots, b_{k}\right)=\mathrm{P}\left(\varphi\left(a_{1}\right)=b_{1}, \ldots, \varphi\left(a_{k}\right)=b_{k}\right)=\mathrm{E} \prod_{i=1}^{k} \mu_{a_{i}}\left(\varphi, b_{i}\right), \\
P\left(a_{1}, a_{2} ; \beta\right)=\sum_{\left\langle b_{1}, b_{2} \in B \mid \llbracket b_{1}, b_{2} \rrbracket=\beta\right\rangle} P\left(a_{1}, a_{2} ; b_{1}, b_{2}\right), \\
P^{\#}(\theta ; \beta)=\frac{1}{|A| N_{\mathcal{L}}(\theta)} \sum_{\left\langle a_{1}, a_{2} \in A \mid \llbracket a_{1}, a_{2} \rrbracket=\theta\right\rangle} P\left(a_{1}, a_{2} ; \beta\right) .
\end{gathered}
$$

Замечание 3.1. При наших ограничениях на выбор левой части неравенство $P^{\#}(\theta ; \beta) \neq 0$ возможно только в случае $\beta \leq \tau(\theta)$.

Чтобы подсчитать среднее число решений уравнения $\Psi$, воспользуемся формулой (2.3). Из нее следует

$$
\mathrm{E} \eta(\Psi)=\frac{\mathrm{E} \eta(\Phi)^{2}}{\mathrm{E} \eta(\Phi)} .
$$

Воспользовавшись равенствами (2.1) и доказанными в работе [6] равенствами

$$
\mathrm{E} \eta\left(\Phi^{\theta}\right)=\frac{|A / \xi(\theta)|}{|B / \zeta(\tau(\theta))|}, \quad \mathrm{E} \eta(\Phi)=\frac{|A|}{|B|},
$$


получаем

$$
\begin{gathered}
\operatorname{E} \eta(\Psi)=\frac{|B|}{|A|} \sum_{f, b} \frac{p(f)}{|B|}\left(\sum_{a \in A} \mu_{a}(f, b)\right)^{2}= \\
=\frac{|B|}{|A|} \sum_{f} \frac{p(f)}{|B|}\left\{\sum_{a_{1}, a_{2} \in A} \sum_{b \in B} \mu_{a_{1}}(f, b) \mu_{a_{2}}(f, b)\right\} .
\end{gathered}
$$

Заметим, что величина в фигурных скобках равна 1 , если $f\left(a_{1}\right)=f\left(a_{2}\right)$, и 0 в противном случае. Отсюда следует

$$
\begin{aligned}
\mathrm{E} \eta(\Psi) & =\frac{1}{|A|} \sum_{a_{1}, a_{2} \in A} P\left(a_{1}, a_{2} ; 0_{\mathcal{M}}\right)=\frac{1}{|A|} \sum_{\rho \in \mathcal{L}} \sum_{\left\langle a_{1}, a_{2} \in A \mid \llbracket a_{1}, a_{2} \rrbracket=\rho\right\rangle} P\left(a_{1}, a_{2} ; 0_{\mathcal{M}}\right)= \\
& =\frac{1}{|A|} \sum_{\rho \in \mathcal{L}}|A| N_{\mathcal{L}}(\rho) P^{\#}\left(\rho ; 0_{\mathcal{M}}\right)=\sum_{\rho \in \mathcal{L}} N_{\mathcal{L}}(\rho) P^{\#}\left(\rho ; 0_{\mathcal{M}}\right) .
\end{aligned}
$$

В принципе, значение $\mathrm{E} \eta\left(\Psi^{\theta}\right)$ можно вычислить напрямую, воспользовавшись равенством (2.6), однако мы выберем другой метод, основанный на следующей лемме, поскольку она может оказаться полезной и в других случаях.

Лемма 3.1. Пусть $\theta \in \mathcal{L}$. Тогда уравнение $\Phi^{\theta}$ является уравнением с независимой, равномерно распределенной правой частью, а уравнение $\Psi^{\theta}$ - заведомо разрешимое, согласованное с уравнением $\Phi^{\theta}$.

Доказательство. Первое утверждение доказано в работе [6].

Рассмотрим какое-либо уравнение $(f, b) \in \mathbb{U}(A, B ; v)$. Напомним, что $(f, b)^{\theta}=\left(f_{\theta \tau(\theta)}, \sigma_{\tau(\theta)}(b)\right)$, где $f_{\theta \tau(\theta)}: A / \xi(\theta) \rightarrow B / \zeta(\tau(\theta))$ - единственное отображение, удовлетворяющее равенству

$$
f_{\theta \tau(\theta)} \pi_{\theta}=\sigma_{\tau(\theta)} f
$$

Пусть $\mathbf{a} \in A / \xi(\theta), a \in A$ и $\pi_{\theta}(a)=\mathbf{a}$. Учитывая равенство (3.7), получаем, что $f_{\theta \tau(\theta)}(\mathbf{a})=\sigma_{\tau(\theta)} f(a)$. Отсюда легко следует, что элемент $\mathbf{a}$ является решением уравнения $(f, b)^{\theta}$ тогда и только тогда, когда элемент $a$ является решением одного из уравнений $\left(f, b^{\prime}\right) \quad\left(b^{\prime} \in \mathcal{S}_{\tau(\theta)}(b)\right)$. При этом элемент $a$ может быть решением только одного из этих уравнений. Значит,

$$
\mu_{\mathrm{a}}\left((f, b)^{\theta}\right)=\sum_{b^{\prime} \in \mathcal{S}_{\mathcal{\tau}}(\theta)(b)} \mu_{a}\left(f, b^{\prime}\right) .
$$

Функции $\mu_{\mathrm{a}}, \mu_{a}$ определяются формулой (2.1). 
Равенство вида (3.8) выполняется для каждого такого элемента $a \in A$, что $\pi_{\theta}(a)=\mathbf{a}$. Просуммировав все такие уравнения, получаем

$$
\left|\pi_{\theta}^{-1}(\mathbf{a})\right| \mu_{\mathbf{a}}\left((f, b)^{\theta}\right)=\sum_{a \in \pi_{\theta}^{-1}(\mathbf{a})} \sum_{b^{\prime} \in \mathcal{S}_{\tau}(\theta)(b)} \mu_{a}\left(f, b^{\prime}\right)
$$

Ясно, что множество $\pi_{\theta}^{-1}(\mathbf{a})$ является одним из классов эквивалентности отношения $\xi(\theta)$. Поэтому $\left|\pi_{\theta}^{-1}(\mathbf{a})\right|=|A||A / \xi(\theta)|^{-1}$ и

$$
\mu_{\mathbf{a}}\left((f, b)^{\theta}\right)=\frac{|A / \xi(\theta)|}{|A|} \sum_{a \in \pi_{\theta}^{-1}(\mathbf{a})} \sum_{b^{\prime} \in \mathcal{S}_{\tau(\theta)}(b)} \mu_{a}\left(f, b^{\prime}\right) .
$$

Таким образом, число решений уравнения $(f, b)^{\theta}$ равно

$$
\begin{aligned}
& \eta\left((f, b)^{\theta}\right)=\sum_{a \in A / \xi(\theta)} \mu_{a}\left((f, b)^{\theta}\right)=\frac{|A / \xi(\theta)|}{|A|} \sum_{a \in A / \xi(\theta)} \sum_{a \in \pi_{\theta}^{-1}(a)} \sum_{b^{\prime} \in \mathcal{S}_{\tau(\theta)}(b)} \zeta_{a}\left(f, b^{\prime}\right)= \\
& =\frac{|A / \xi(\theta)|}{|A|} \sum_{b^{\prime} \in \mathcal{S}_{\tau(\theta)}(b)} \sum_{a \in A} \mu_{a}\left(f, b^{\prime}\right)=\frac{|A / \xi(\theta)|}{|A|} \sum_{b^{\prime} \in \mathcal{S}_{\tau(\theta)}(b)} \eta\left(f, b^{\prime}\right) .
\end{aligned}
$$

Пусть $h$ - функция, заданная на множестве $\mathbb{U}\left(A / \theta, B / \tau(\theta) ; v^{\theta}\right)$. Тогда

$$
\begin{gathered}
E h\left(\Phi^{\theta}\right) \eta\left(\Phi^{\theta}\right)=\sum_{f, b} p(f) \frac{1}{|B|} h\left((f, b)^{\theta}\right) \frac{|A / \xi(\theta)|}{|A|} \sum_{b^{\prime} \in \mathcal{S}_{\tau(\theta)}(b)} \eta\left(f, b^{\prime}\right)= \\
=\frac{|A / \xi(\theta)|}{|A|} \frac{1}{|B|} \sum_{b^{\prime} \in B} \sum_{f} p(f) \eta\left(f, b^{\prime}\right) \sum_{b \in \mathcal{S}_{\tau(\theta)}\left(b^{\prime}\right)} h\left((f, b)^{\theta}\right) .
\end{gathered}
$$


Непосредственно из определений следует, что $(f, b)^{\theta}=\left(f, b^{\prime}\right)^{\theta}$ для всех $\left(b, b^{\prime}\right) \in \tau(\theta)$. Поэтому

$$
\sum_{b \in \mathcal{S}_{\left.\tau(\theta)^{(}\right)}\left(b^{\prime}\right)} h\left((f, b)^{\theta}\right)=\left|\mathcal{S}_{\tau(\theta)}\left(b^{\prime}\right)\right| h\left(\left(f, b^{\prime}\right)^{\theta}\right)=\frac{|B|}{|B / \zeta(\tau(\theta))|} h\left(\left(f, b^{\prime}\right)^{\theta}\right) .
$$

Подставив полученное выражение в формулу (3.10), получаем

$$
\begin{gathered}
\mathrm{E}\left(h\left(\Phi^{\theta}\right) \eta\left(\Phi^{\theta}\right)\right)=\frac{|A / \xi(\theta)|}{|A|} \frac{1}{|B|} \sum_{b^{\prime} \in B} \sum_{f} p(f) \eta\left(f, b^{\prime}\right) \frac{|B|}{|B / \zeta(\tau(\theta))|} h\left(\left(f, b^{\prime}\right)^{\theta}\right)= \\
=\frac{|A / \xi(\theta)|}{|A|}\left|\frac{|B|}{|B / \zeta(\tau(\theta))|}\right| \frac{1}{|B|} \sum_{b^{\prime} \in B} \sum_{f} p(f) \eta\left(f, b^{\prime}\right) h\left(\left(f, b^{\prime}\right)^{\theta}\right)= \\
=\frac{|A / \xi(\theta)|}{|B / \zeta(\tau(\theta))|} \mid \frac{|B|}{|A|} \mathrm{E}\left(h\left(\Phi^{\theta}\right) \eta(\Phi)\right)=\mathrm{E} \eta\left(\Phi^{\theta}\right) \frac{\mathrm{E}\left(h\left(\Phi^{\theta}\right) \eta(\Phi)\right)}{\mathrm{E} \eta(\Phi)}= \\
=\mathrm{E} \eta\left(\Phi^{\theta}\right) \mathrm{E} h\left(\Psi^{\theta}\right) .
\end{gathered}
$$

В последних равенствах мы воспользовались формулами (3.5) и (2.6). Таким образом, имеет место равенство $\mathrm{E} h\left(\Psi^{\theta}\right)=\frac{\mathrm{E} h\left(\Phi^{\theta}\right) \eta\left(\Phi^{\theta}\right)}{\mathrm{E} \eta\left(\Phi^{\theta}\right)}$. Из него так же, как при доказательстве теоремы 2.1, выводим утверждение о согласованности уравнений.

Лемма 3.1 позволяет доказывать утверждения о факторуравнениях только для исходных уравнений.

Теорема 3.1. Пусть $\Phi-$ случайное уравнение с независимой, равномерно распределенной правой частью, $\Psi$ - согласованное с ним заведомо разрешимое уравнение. Тогда для каждого элемента $\theta \in \mathcal{L}$ выполняется 
неравенство $\mathrm{E} \xi\left(\Psi^{\theta}\right) \geq \frac{\mathrm{E} \xi\left(\Phi^{\theta}\right)}{q_{\mathrm{нр}}^{\theta}}$, где $q_{\mathrm{Hр}}^{\theta}-$ вероятность неразрешимости уравнения $\Phi^{\theta}$.

Неравенство $\mathrm{E} \xi(\Psi) \geq \frac{\mathrm{E} \xi(\Phi)}{q_{\text {нр }}}$, где $q_{\text {нр }}$ - вероятность неразрешимости уравнения $\Phi$, доказывается так же, как лемма 4.3 работы [1]. Применив лемму 3.1 , получаем требуемое неравенство.

В частности, данное утверждение показывает, что средняя трудоемкость решения заведомо разрешимого уравнения переборными методами всегда больше или равна средней трудоемкости решения исходного случайного уравнения.

Вернемся к вычислению величины $\mathrm{E}\left(\Psi^{\theta}\right)$. Дословно повторяя доказательство формулы (3.9), получаем

$$
\begin{gathered}
\sum_{\mathbf{a}_{1}, \mathbf{a}_{2} \in A / \xi(\theta)} P^{\theta}\left(\mathbf{a}_{1}, \mathbf{a}_{2} ; \tau(\theta)\right)=\sum_{\substack{\mathbf{a}_{1}, \mathbf{a}_{2} \in A / \xi(\theta) \\
\mathbf{b} \in B / \zeta(\tau(\theta))}} \mathrm{P}\left(\varphi^{\theta}\left(\mathbf{a}_{1}\right)=\mathbf{b}, \varphi^{\theta}\left(\mathbf{a}_{2}\right)=\mathbf{b}\right)= \\
=\left(\left.\frac{|A / \xi(\theta)|}{|A|}\right|_{\substack{a_{1}, a_{2} \in A \\
\left\langle b_{1}, b_{2} \in B \mid \llbracket b_{1}, b_{2} \rrbracket \leq \tau(\theta)\right\rangle}} \mathrm{P}\left(\varphi\left(a_{1}\right)=b_{1}, \varphi\left(a_{2}\right)=b_{2}\right)=\right. \\
=\left(\left.\frac{|A / \xi(\theta)|}{|A|}\right|^{2} \sum_{a_{1}, a_{2} \in A} \sum_{f} p(f) \sum_{\left\langle b_{1}, b_{2} \in B \mid \llbracket b_{1}, b_{2} \rrbracket \leq \tau(\theta)\right\rangle} \mu_{a_{1}}\left(f, b_{1}\right) \mu_{a_{2}}\left(f, b_{2}\right) .\right.
\end{gathered}
$$

Заметим, что среди слагаемых, входящих в последнюю сумму, ненулевым может быть только один, причем такой ненулевой член имеется тогда и только тогда, когда $\llbracket f\left(a_{1}\right), f\left(a_{2}\right) \rrbracket \leq \tau(\theta)$. Поэтому

$$
\begin{gathered}
\sum_{f} p(f) \sum_{\left\langle b_{1}, b_{2} \in B \mid \llbracket b_{1}, b_{2} \| \leq \tau(\theta)\right\rangle} \mu_{a_{1}}\left(f, b_{1}\right) \mu_{a_{2}}\left(f, b_{2}\right)= \\
=\sum_{\left\langle f \|\left[f\left(a_{1}\right), f\left(a_{2}\right) \| \leq \tau(\theta)\right\rangle\right.} p(f)=\sum_{\beta \leq \tau(\theta)} P\left(a_{1}, a_{2} ; \beta\right) .
\end{gathered}
$$


Таким образом,

$$
\sum_{\mathbf{a}_{1}, \mathbf{a}_{2} \in A / \xi(\theta)} P^{\theta}\left(\mathbf{a}_{1}, \mathbf{a}_{2} ; \Delta_{B / \tau(\theta)}\right)=\left(\frac{|A / \xi(\theta)|}{|A|}\right)^{2} \sum_{a_{1}, a_{2} \in A} \sum_{\beta \leq \tau(\theta)} P\left(a_{1}, a_{2} ; \beta\right) .
$$

Теперь из равенства (3.6) и леммы 3.1 следует, что

$$
\begin{gathered}
\operatorname{E} \eta\left(\Psi^{\theta}\right)=|A / \xi(\theta)|^{-1} \sum_{\mathbf{a}_{1}, \mathbf{a}_{2} \in A / \xi(\theta)} P^{\theta}\left(\mathbf{a}_{1}, \mathbf{a}_{2} ; \Delta_{B / \zeta(\tau(\theta))}\right)= \\
=\frac{|A / \xi(\theta)|}{|A|^{2}} \sum_{a_{1}, a_{2} \in A} \sum_{\beta \leq \tau(\theta)} P\left(a_{1}, a_{2} ; \beta\right)= \\
=\frac{|A / \xi(\theta)|}{|A|^{2}} \sum_{\rho \in \mathcal{L}}|A| N_{\mathcal{L}}(\rho) \sum_{\beta \leq \tau(\theta)} P^{\#}(\rho ; \beta)=\frac{|A / \xi(\theta)|}{|A|} \sum_{\rho \in \mathcal{L}} N_{\mathcal{L}}(\rho) \sum_{\beta \leq \tau(\theta)} P^{\#}(\rho ; \beta) .
\end{gathered}
$$

Дальнейшее исследования требуют конкретизации функции $P^{\#}$. Укажем три их естественных типа.

Случайное отображение назовем инъективным, если каждое отображение $f$, для которого $p(f)>0$, инъективно. Нетрудно видеть, что инъективность случайного отображения эквивалентна условию

$$
\left(\theta>0_{\mathcal{L}}\right) \Rightarrow\left(P^{\#}\left(\theta ; 0_{\mathcal{L}}\right)=0\right) .
$$

Ясно, что если левая часть случайного уравнения инъективна, то это уравнение не может иметь более одного решения. В частности, для заведомо совместного уравнения число решений всегда равно 1. Если инъективными являются все отображения $f_{\theta \tau(\theta)}$, то случайное отображение $\varphi$ назовем наследственно инъективныл. Это свойство определяется условием

$$
(\rho>\theta, \beta<\tau(\theta)) \Rightarrow\left(P^{\#}(\rho ; \beta)=0\right) .
$$

В этом случае каждое факторуравнение $\Psi^{\theta}$ также имеет ровно одно решение.

Противоположным является случай вырожденного отображения, при котором $p(f)>0$ только для таких отображений $f$, что $|f(A)|=1$. Этот случай определяется условием

$$
\left(\beta>0_{\mathcal{M}}\right) \Rightarrow\left(P^{\#}(\theta ; \beta)=0\right) .
$$


Ясно, что для него $\mathrm{E} \Psi^{\theta}=|A / \theta|$. Этот случай практического интереса не имеет и приведен только для полноты изложения.

Прежде чем определить еще один случай, проведем некоторые рассмотрения. В криптографических приложениях, как правило, используются случайные отображения $\varphi$, значения которых $\varphi\left(a_{1}\right), \varphi\left(a_{2}\right)$ на различных элементах $a_{1}, a_{2} \in A$ независимы или слабо зависимы. Числовую меру зависимости можно выбирать различными способами. В частности, ее можно измерять условной энтропией случайного элемента $\varphi\left(a_{2}\right)$ относительно случайного элемента $\varphi\left(a_{1}\right)$ :

$$
H\left(a_{2} \mid a_{1}\right)=-\sum_{b_{1} \in B} \sum_{b_{2} \in B} P\left(a_{1}, a_{2} ; b_{1}, b_{2}\right) \log _{2} \frac{P\left(a_{1}, a_{2} ; b_{1}, b_{2}\right)}{P\left(a_{1} ; b_{1}\right)} .
$$

Желательно, чтобы эта величина была максимальной.

Допустим, что $\llbracket a_{1}, a_{2} \rrbracket=\theta$. Тогда при наших ограничениях на случайное отображение $\varphi$ величина $P\left(a_{1}, a_{2} ; b_{1}, b_{2}\right)$ может быть ненулевой только при $\llbracket b_{1}, b_{2} \rrbracket \leq \tau(\theta)$. Поэтому

$$
\begin{aligned}
& -\sum_{b_{2} \in B} P\left(a_{1}, a_{2} ; b_{1}, b_{2}\right) \log _{2} \frac{P\left(a_{1}, a_{2} ; b_{1}, b_{2}\right)}{P\left(a_{1} ; b_{1}\right)}= \\
= & -\sum_{b_{2} \in \mathcal{S}_{\mathcal{\tau}(\theta)}\left(b_{1}\right)} P\left(a_{1}, a_{2} ; b_{1}, b_{2}\right) \log _{2} \frac{P\left(a_{1}, a_{2} ; b_{1}, b_{2}\right)}{P\left(a_{1} ; b_{1}\right)} .
\end{aligned}
$$

Хорошо известно, что максимальное значение суммы из правой части равенства достигается, если все входящие в нее величины $P\left(a_{1}, a_{2} ; b_{1}, b_{2}\right)$ равны. Так как $\sum_{b_{2} \in B} P\left(a_{1}, a_{2} ; b_{1}, b_{2}\right)=P\left(a_{1} ; b_{1}\right)$, то максимальное значение суммы достигается в случае

$$
P\left(a_{1}, a_{2} ; b_{1}, b_{2}\right)=\left\{\begin{array}{cl}
P\left(a_{1} ; b_{1}\right)|B / \tau(\theta)||B|^{-1}, & \llbracket b_{1}, b_{2} \rrbracket \leq \tau\left(\llbracket a_{1}, a_{2} \rrbracket\right) ; \\
0, & \llbracket b_{1}, b_{2} \rrbracket
\end{array}\right.
$$

Случайное отображение $\varphi$, для которого эти равенства выполняются для всех элементов $a_{i}, b_{j}$, будем называть слабо коррелированным. Важный для 
практики пример слабо коррелированного отображения будет построен в следующем разделе.

Пусть $a_{1}, a_{2} \in A$ и $\llbracket a_{1}, a_{2} \rrbracket=\theta$. Если $\beta \leq \tau(\theta)$, то для слабо коррелированного отображения имеем

$$
\begin{gathered}
\sum_{\left\langle b_{1}, b_{2} \in B \mid \llbracket b_{1}, b_{2} \rrbracket=\beta\right\rangle} P\left(a_{1}, a_{2} ; b_{1}, b_{2}\right)=\sum_{b_{1} \in B} \sum_{b_{2} \in \mathcal{C}_{\beta}\left(b_{1}\right)} P\left(a_{1} ; b_{1}\right)|B / \zeta(\tau(\theta))||B|^{-1}= \\
=\left.\sum_{b_{1} \in B} P\left(a_{1} ; b_{1}\right)|B / \zeta(\tau(\theta))| B\right|^{-1} N_{\mathcal{M}}(\beta)=\frac{|B / \zeta(\tau(\theta))| N_{\mathcal{M}}(\beta)}{|B|} .
\end{gathered}
$$

Таким образом,

$$
P^{\#}(\theta ; \beta)=\left\{\begin{array}{cc}
\frac{|B / \tau(\theta)|^{N \mathcal{M}(\beta)}}{|B|}, & \beta \leq \tau(\theta) ; \\
0, & \beta \notin \tau(\theta) .
\end{array}\right.
$$

Можно ожидать, что функции именно такого вида будут чаще всего встречаться в криптографических приложениях.

Подставив выражения (3.13) в формулу (3.12), получаем формулу для вычисления среднего числа решений в случае слабо коррелированного отображения:

$$
\mathrm{E} \eta\left(\Psi^{\theta}\right)=\frac{|A / \xi(\theta)|}{|A|} \sum_{\rho \in \mathcal{L}} N_{\mathcal{L}}(\rho) \sum_{\beta \leq \tau(\theta) \wedge \tau(\rho)} \frac{|B / \zeta(\tau(\rho))| N_{\mathcal{M}}(\beta)}{|B|} .
$$

Для упрощения вычислений можно использовать асимптотические оценки. Для их получения рассмотрим последовательности $\left\{A_{n}\right\}_{n \in \mathbb{N}},\left\{B_{n}\right\}_{n \in \mathbb{N}}$ конечных множеств и последовательности перестановочных, однородных представлений $\xi_{n}: \mathcal{L} \rightarrow \mathcal{E}\left(A_{n}\right), \zeta_{n}: \mathcal{M} \rightarrow \mathcal{E}\left(B_{n}\right)$ фиксированных решеток $\mathcal{L}, \mathcal{M}$.

Наличие представлений позволяет определить на каждом из множеств $A_{n}, B_{n}$ расстояния $\llbracket \cdot, \cdot \rrbracket$, а это позволяет определить сферы, т. е. подмножества вида $\left\langle a^{\prime} \in A_{n}=\mid \llbracket a, a^{\prime} \rrbracket=\theta\right\rangle$ и $\left\langle b^{\prime} \in B_{n}=\mid \llbracket b, b^{\prime} \rrbracket=\beta\right\rangle$. Числа элементов указанных сфер обозначим через $N_{n \mathcal{L}}(\theta)$ и $N_{n \mathcal{M}}(\beta)$ соответственно.

Теперь рассмотрим последовательность $\left\{\Phi_{n}\right\}_{n \in \mathbb{N}}$ случайных уравнений c независимой правой частью, принимающих значения в множествах 
$\mathbb{U}\left(A_{n}, B_{n} ; v\right)$. По аналогии с величиной (3.3) для каждого номера $n$ определяем величину $P_{n}^{\#}(\rho ; \beta)$.

Пусть $\Psi_{n}$ - заведомо разрешимое уравнение, согласованное со случайным уравнением $\Phi_{n}$.

Теорема 3.2. Пусть

$$
\lim _{n \rightarrow \infty}\left|A_{n} / \xi_{n}(\rho)\right|\left|A_{n} / \xi_{n}(\theta)\right|^{-1}=\infty, \lim _{n \rightarrow \infty}\left|B_{n} / \zeta_{n}(\alpha)\right|\left|B_{n} / \zeta_{n}(\beta)\right|^{-1}=\infty
$$

для всех отношений $\rho<\theta, \alpha<\beta$ и

$$
\lim _{n \rightarrow \infty} \frac{\left|B_{n}\right|}{\left|B_{n} / \zeta_{n}(\tau(\rho))\right| N_{n} \mathcal{M}^{(\beta)}} P_{n}^{\#}(\rho ; \beta)=1
$$

при всех таких $\rho \in \mathcal{L}, \beta \in \mathcal{M}$, что $\beta \leq \tau(\rho)$. Тогда

$$
\mathrm{E} \eta\left(\Psi_{n}^{\theta}\right)=(1+o(1)) \frac{\left|A_{n} / \xi_{n}(\theta)\right|}{\left|B_{n} / \zeta_{n}(\tau(\theta))\right|} \sum_{\langle\rho \in \mathcal{L} \mid \rho \geq \theta\rangle} \frac{\left|B_{n} / \zeta_{n}(\tau(\rho))\right|}{\left|A_{n} / \xi_{n}(\rho)\right|} .
$$

Из формулы (3.2) и сделанных предположений следует, что

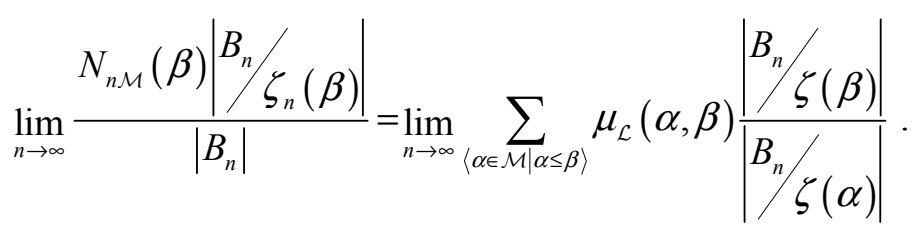

Вычислим правую часть.

По свойствам функции Мёбиуса выполняется равенство $\mu_{\mathcal{M}}(\beta, \beta)=1$. Из него и предположений теоремы следует, что для каждого элемента $\alpha \in \mathcal{M}$, меньшего или равного элементу $\beta$, выполняется равенство

$$
\lim _{n \rightarrow \infty} \mu_{\mathcal{M}}(\alpha, \beta) \frac{\left|B_{n} / \zeta_{n}(\beta)\right|}{\left|B_{n} / \zeta_{n}(\alpha)\right|}= \begin{cases}1, & \alpha=\beta ; \\ 0, & \alpha<\beta .\end{cases}
$$


Так как множество $\langle\alpha \in \mathcal{M} \mid \alpha \leq \beta\rangle$ конечно, то

Таким образом,

$$
1=\sum_{\langle\alpha \in \mathcal{M} \mid \alpha \leq \beta\rangle} \lim _{n \rightarrow \infty} \mu_{\mathcal{M}}(\alpha, \beta) \frac{\left|B_{n} / \zeta(\beta)\right|}{\left|B_{n} / \zeta(\alpha)\right|}=\lim _{n \rightarrow \infty} \sum_{\langle\alpha \in \mathcal{M} \mid \alpha \leq \beta\rangle} \mu_{\mathcal{M}}(\alpha, \beta) \frac{\left|B_{n} / \zeta(\beta)\right|}{\left|B_{n} / \zeta(\alpha)\right|} .
$$

$$
\lim _{n \rightarrow \infty} \frac{N_{n \mathcal{M}}(\beta)\left|B_{n} / \zeta_{n}(\beta)\right|}{\left|B_{n}\right|}=1 .
$$

По аналогии с доказательством следствия 2.1 работы [6] можно показать, что из перестановочности и однородности решетки $\mathcal{M}$ вытекает равенство

$$
\left|B_{n} / \zeta_{n}(\tau(\theta) \wedge \tau(\rho))\right|\left|B_{n} / \zeta_{n}(\tau(\theta) \vee \tau(\rho))\right|=\left|B_{n} / \zeta_{n}(\tau(\theta))\right|\left|B_{n} / \zeta_{n}(\tau(\rho))\right|,
$$

а из него и того, что отображение $\tau$ является верхним гомоморфизмом, следует равенство

$$
\frac{\left|B_{n} / \zeta_{n}(\tau(\theta) \wedge \tau(\rho))\right|}{\left|B_{n} / \zeta_{n}(\tau(\rho))\right|}=\frac{\left|B_{n} / \zeta_{n}(\tau(\theta))\right|}{\left|B_{n} / \zeta_{n}(\tau(\theta) \vee \tau(\rho))\right|}=\frac{\left|B_{n} / \zeta_{n}(\tau(\theta))\right|}{\left|B_{n} / \zeta_{n}(\tau(\theta \vee \rho))\right|} .
$$

$\mathrm{B}$ силу равенств (3.15), (3.16) для каждого элемента $\beta$, меньшего или равного $\tau(\theta) \wedge \tau(\rho)$, выполняется равенство

$$
\begin{aligned}
& \lim _{n \rightarrow \infty} \frac{\left|B_{n} / \zeta_{n}(\tau(\theta))\right|}{\left|B_{n} / \zeta_{n}(\tau(\theta \vee \rho))\right|} P_{n}^{\#}(\rho ; \beta)=\lim _{n \rightarrow \infty} \frac{\left|B_{n} / \zeta_{n}(\tau(\theta) \wedge \tau(\rho))\right|}{\left|B_{n} / \zeta_{n}(\tau(\rho))\right|} P_{n}^{\#}(\rho ; \beta)= \\
& =\lim _{n \rightarrow \infty}\left(\frac{\left|B_{n} / \zeta_{n}(\tau(\theta) \wedge \tau(\rho))\right|}{\left|B_{n} / \zeta_{n}(\rho)\right|}\right) \mid\left(\frac{\left|B_{n} / \zeta_{n}(\rho)\right| N_{n \mathcal{M}}(\beta)}{\left|B_{n}\right|}\right) \times \\
& \times\left(\frac{\left|B_{n}\right|}{\left|B_{n} / \zeta_{n}(\tau(\rho))\right| N_{n \mathcal{M}}(\beta)} P_{n}^{\#}(\rho ; \beta)\right)= \begin{cases}1, & \rho=\tau(\theta) \wedge \tau(\rho) ; \\
0, & \rho<\tau(\theta) \wedge \tau(\rho) .\end{cases}
\end{aligned}
$$


Просуммировав все полученные равенства и воспользовавшись тем, что область суммирования конечна и не зависит от номера $n$, получаем

$$
\lim _{n \rightarrow \infty} \frac{\left|B_{n} / \zeta_{n}(\tau(\theta))\right|}{\left|B_{n} / \zeta_{n}(\tau(\theta \vee \rho))\right|} \sum_{\beta \leq \tau(\theta) \wedge \tau(\rho)} P_{n}^{\#}(\rho ; \beta)=1 .
$$

По аналогии с равенством (3.16) показываем, что

$$
\lim _{n \rightarrow \infty} \frac{N_{n \mathcal{L}}(\rho)\left|A_{n} / \rho\right|}{\left|A_{n}\right|}=1 .
$$

Из доказанных равенств и формулы (3.14) следует соотношение

$$
\begin{aligned}
& E \eta\left(\Psi_{n}^{\theta}\right)=\left|A_{n} / \xi_{n}(\theta)\right| \sum_{\rho \in \mathcal{L}} \frac{1}{\left|A_{n} / \xi_{n}(\rho)\right|} \frac{\left|B_{n} / \zeta_{n}(\tau(\theta \vee \rho))\right|}{\left|B_{n} / \zeta_{n}(\tau(\theta))\right|}(1+o(1))= \\
& =\frac{\left|A_{n} / \xi_{n}(\theta)\right|}{\left|B_{n} / \zeta_{n}(\tau(\theta))\right|}\left\{\sum_{\rho \geq \theta} \frac{\left|B_{n} / \zeta_{n}(\tau(\rho))\right|}{\left|A_{n} / \xi_{n}(\rho)\right|}(1+o(1))+\right. \\
& \left.+\sum_{\rho \ngtr \theta} \frac{\left|B_{n} / \zeta_{n}(\tau(\theta \vee \rho))\right|\left|\frac{A_{n} / \xi_{n}(\theta \vee \rho) \mid}{\left|A_{n} / \xi_{n}(\theta \vee \rho)\right|}\right|}{\left|A_{n} / \xi_{n}(\rho)\right|}(1+o(1))\right\}= \\
& =\frac{\left|A_{n} / \xi_{n}(\theta)\right|}{\left|B_{n} / \zeta_{n}(\tau(\theta))\right|} \sum_{\omega \geq \theta} \frac{\left|B_{n} / \zeta_{n}(\tau(\omega))\right|}{\left|A_{n} / \xi_{n}(\omega)\right|}\left(1+o(1)+\sum_{\substack{\rho \ngtr \theta \\
\rho \vee \theta=\omega}} \frac{\left|A_{n} / \xi_{n}(\omega)\right|}{\left|A_{n} / \xi_{n}(\rho)\right|}(1+o(1))\right),
\end{aligned}
$$

что и доказывает утверждение теоремы. 
Отметим, что если все случайные уравнения $\Phi_{n}$ слабо коррелированны, то $\frac{\left|B_{n}\right|}{\left|B_{n} / \zeta_{n}(\tau(\rho))\right| N_{n} \mathcal{M}^{(\beta)}} P_{n}^{\#}(\rho ; \beta)$ для всех таких элементов $\rho, \beta$, что $\beta \leq \tau(\rho)$, и равенство (3.15) выполняется.

Асимптотическая формула может быть еще упрощена. Для этого воспользуемся введенной в работе [6] величиной $d_{n}(\theta)=\min _{\langle\rho \in \mathcal{L} \mid \rho \geq \theta\rangle} \ln \frac{\left|A_{n} / \xi_{n}(\rho)\right|}{\left|B_{n} / \zeta_{n}(\tau(\rho))\right|}$. Через $\delta_{n}(\theta)$ обозначим один из элементов, на котором достигается минимальное значение. Используя введенные обозначения, получаем

$$
\begin{gathered}
e^{d_{n}(\theta)} \sum_{\langle\rho \in \mathcal{L} \mid \rho \geq \theta\rangle} \frac{\left|B_{n} / \zeta_{n}(\tau(\rho))\right|}{\left|A_{n} / \xi_{n}(\rho)\right|}=\sum_{\langle\rho \in \mathcal{L} \mid \rho \geq \theta\rangle} \frac{\left|B_{n} / \zeta_{n}(\tau(\rho))\right|}{\left|A_{n} / \xi_{n}(\rho)\right|}\left|\frac{\left|B_{n} / \zeta_{n}\left(\tau\left(\delta_{n}(\theta)\right)\right)\right|}{\left|A_{n} / \xi_{n}\left(\delta_{n}(\theta)\right)\right|}\right|^{-1}= \\
=1+\sum_{\left.\langle\rho \in \mathcal{L}| \begin{array}{c}
\rho \geq \theta \\
\left.\rho \neq \delta_{n}(\theta)\right\rangle
\end{array}\right)} \frac{\left|B_{n} / \zeta_{n}(\tau(\rho))\right|}{\left|A_{n} / \xi_{n}(\rho)\right|} \mid \frac{\left|B_{n} / \zeta_{n}\left(\tau\left(\delta_{n}(\theta)\right)\right)\right|}{\left|A_{n} / \xi_{n}\left(\delta_{n}(\theta)\right)\right|} .
\end{gathered}
$$

Из определений следует, что каждое слагаемое в правой части больше или равно 0 и меньше или равно 1 . Поэтому

$$
1 \leq e^{d_{n}(\theta)} \sum_{\langle\rho \in \mathcal{L} \mid \rho \geq \theta\rangle} \frac{\left|B_{n} / \zeta_{n}(\tau(\rho))\right|}{\left|A_{n} / \xi_{n}(\rho)\right|} \leq C(\theta)
$$


где $C(\theta)$ - мощность множества $\langle\rho \in \mathcal{L} \mid \rho \geq \theta\rangle$. Теперь из доказанной теоремы следует, что

$$
\begin{aligned}
& \mathrm{E} \eta\left(\Psi_{n}^{\theta}\right)=\Gamma_{n}(\theta) \frac{\left|A_{n} / \xi_{n}(\theta)\right|}{\left|B_{n} / \zeta_{n}(\tau(\theta))\right|} e^{-d_{n}(\theta)}, \\
& 1 \leq \varliminf_{n \rightarrow \infty} \Gamma_{n}(\theta), \quad \frac{\lim _{n \rightarrow \infty} \Gamma_{n}(\theta) \leq C(\theta) .}{}
\end{aligned}
$$

Следствие 3.1. Пусть выполнены предположения теоремы 3.2. Тогда трудоемкости решения уравнений $\Phi_{n} u \Psi_{n}$ связаны неравенствами

$$
\varliminf_{n \rightarrow \infty} \frac{T\left(\Psi_{n}\right)}{T\left(\Phi_{n}\right)} \geq 1, \quad \varlimsup_{n \rightarrow \infty} \frac{T\left(\Psi_{n}\right)}{T\left(\Phi_{n}\right) e^{-d_{n}\left(0_{\mathcal{L}}\right)}} \leq|\mathcal{L}| .
$$

Заметим, что

$$
\begin{aligned}
& d_{n}(\theta)=\min _{\langle\rho \in \mathcal{L} \mid \rho \geq \theta\rangle} \ln \frac{\left|A_{n} / \xi_{n}(\rho)\right|}{\left|B_{n} / \zeta_{n}(\tau(\rho))\right|} \leq \min _{\rho \in \mathcal{L}} \ln \frac{\left|A_{n} / \xi_{n}(\rho)\right|}{\left|B_{n} / \zeta_{n}(\tau(\rho))\right|}=d_{n}\left(0_{\mathcal{L}}\right), \\
& d_{n}(\theta)=\min _{\langle\rho \in \mathcal{L} \mid \rho \geq \theta\rangle} \ln \frac{\left|A_{n} / \xi_{n}(\rho)\right|}{\left|B_{n} / \zeta_{n}(\tau(\rho))\right|} \leq \ln \frac{\left|A_{n} / \xi_{n}\left(1_{\mathcal{L}}\right)\right|}{\left|B_{n} / \zeta_{n}\left(\tau\left(1_{\mathcal{L}}\right)\right)\right|}= \\
& =\ln \frac{\left|A_{n} / \nabla_{A n}\right|}{\left|B_{n} / \zeta_{n}\left(\tau\left(1_{\mathcal{L}}\right)\right)\right|}=\ln \frac{1}{\left|B_{n} / \zeta_{n}\left(\tau\left(1_{\mathcal{L}}\right)\right)\right|} \leq 0 .
\end{aligned}
$$

Отсюда и из формул (3.5) и (3.17) следует, что

$$
\begin{gathered}
\varliminf_{n \rightarrow \infty} \frac{\mathrm{E} \eta\left(\Psi_{n}^{\theta}\right)}{\mathrm{E} \eta\left(\Phi_{n}^{\theta}\right)}=\varliminf_{n \rightarrow \infty} \Gamma_{n}(\theta) e^{-d_{n}(\theta)} \geq 1, \\
\varlimsup_{n \rightarrow \infty} \frac{\mathrm{E} \eta\left(\Psi_{n}^{\theta}\right)}{\mathrm{E} \eta\left(\Phi_{n}^{\theta}\right)}=\varliminf_{n \rightarrow \infty} \Gamma_{n}(\theta) e^{-d_{n}(\theta)} \leq C(\theta) e^{-d_{n}\left(0_{\mathcal{L}}\right)} \leq|\mathcal{L}| e^{-d_{n}\left(0_{\mathcal{L}}\right)} .
\end{gathered}
$$


Теперь, применив равенство (3.1), получаем, что для любой последовательности $\left\{\vec{\theta}_{n}\right\}_{n \in \mathbb{N}}$ цепей выполняются неравенства

$$
\varliminf_{n \rightarrow \infty} \frac{T\left(\Psi_{n} ; \overrightarrow{\theta_{n}}\right)}{T\left(\Phi_{n} ; \overrightarrow{\theta_{n}}\right)} \geq 1, \quad \varlimsup_{n \rightarrow \infty} \frac{T\left(\Psi_{n} ; \overrightarrow{\theta_{n}}\right)}{T\left(\Phi_{n} ; \overrightarrow{\theta_{n}}\right) e^{-d_{n}\left(0_{\mathcal{L}}\right)}} \leq|\mathcal{L}| .
$$

Выберем в качестве $\overrightarrow{\theta_{n}}$ цепь решетки $\mathcal{L}$, на которой достигается минимальное значение средней трудоемкости $T\left(\Phi_{n} ; \vec{\theta}\right)$. Тогда

$$
\begin{gathered}
T\left(\Phi_{n} ; \vec{\theta}_{n}\right)=T\left(\Phi_{n}\right), \quad T\left(\Psi_{n} ; \vec{\theta}_{n}\right) \geq T\left(\Psi_{n}\right), \\
\varlimsup_{n \rightarrow \infty} \frac{T\left(\Psi_{n}\right)}{T\left(\Phi_{n}\right) e^{-d_{n}\left(0_{\mathcal{L}}\right)}} \leq \varlimsup_{n \rightarrow \infty} \frac{T\left(\Psi_{n} ; \overrightarrow{\theta_{n}}\right)}{T\left(\Phi_{n} ; \overrightarrow{\theta_{n}}\right) e^{-d_{n}\left(0_{\mathcal{L}}\right)}} \leq|\mathcal{L}| .
\end{gathered}
$$

Теперь выберем в качестве $\overrightarrow{\theta_{n}}$ цепь решетки $\mathcal{L}$, на которой достигается минимальное значение средней трудоемкости $T\left(\Psi_{n} ; \vec{\theta}\right)$. Тогда

$$
\begin{gathered}
T\left(\Phi_{n} ; \vec{\theta}_{n}\right) \geq T\left(\Phi_{n}\right), \quad T\left(\Psi_{n} ; \vec{\theta}_{n}\right)=T\left(\Psi_{n}\right), \\
\underline{\lim _{n \rightarrow \infty}} \frac{T\left(\Psi_{n}\right)}{T\left(\Phi_{n}\right) e^{-d_{n}\left(0_{\mathcal{L}}\right)}} \geq \underline{\lim _{n \rightarrow \infty}} \frac{T\left(\Psi_{n} ; \vec{\theta}_{n}\right)}{T\left(\Phi_{n} ; \vec{\theta}_{n}\right)} \geq 1 .
\end{gathered}
$$

Следствие 3.2. Пусть выполнены предположения следствия 3.1 и для решения заведомо разрешимого уравнения $\Psi_{n}$ используется алгоритм, оnтимизированный для решения случайного уравнения $\Phi_{n}, T^{\prime}\left(\Psi_{n}\right)-$ средняя трудоемкость решения одного уравнения. Тогда неравенства (3.18) останутся справедливыми после замены величины $T\left(\Psi_{n}\right)$ величиной $T^{\prime}\left(\Psi_{n}\right)$.

Утверждение вытекает из доказательства следствия 3.1.

\section{4. Заведомо совместные системы уравнений}

В настоящем разделе будем рассматривать случай, когда множество $B$ разлагается в декартово произведение $B=\prod_{i=1}^{m} B_{i}$. В этом случае каждое отображение $f: A \rightarrow \prod_{i=1}^{m} B_{i}$ однозначно представимо в виде $f=(\underset{i=1}{\mathrm{X}}) f_{i} \quad$ для 
подходящих отображений $f_{i} \in B_{i}^{A}$. Таким образом, уравнение $(f, b) \in \mathbb{U}(A, B)$ эквивалентно системе уравнений

$$
\left\{\begin{array}{c}
f_{1}(x)=b_{1}, \\
f_{2}(x)=b_{2}, \\
\cdots \\
f_{m}(x)=b_{m},
\end{array}\right.
$$

где $\left(f_{i}, b_{i}\right) \in \mathbb{U}\left(A, B_{i}\right)$.

В качестве решетки $\mathcal{M}$ возьмем решетку конгруэнций декартова произведения [6]. Другими словами, решетка $\mathcal{M}$ состоит из всех отношений $\alpha_{J} \quad(J \subseteq\{1,2, \ldots, m\})$, для которых $(\mathbf{b}, \mathbf{c}) \in \alpha_{J}$ тогда и только тогда, когда $b_{j}=c_{j}$ для всех $j \in J$. В качестве представления $\zeta$ возьмем вложение подрешетки $\mathcal{M}$ в $\mathcal{E}(B)$, т. е. $\zeta\left(\alpha_{J}\right)=\alpha_{J}$.

Как мы отмечали выше, фактор-множество $B / \alpha_{J}$ и естественное отображение на него могут быть выбраны произвольно из класса изоморфных объектов. Воспользовавшись этим, выберем в качестве фактормножества множество $B_{J}=\prod_{j \in J} B_{j}$. Если $J=\left\{j_{1}, j_{2}, \ldots, j_{k}\right\}$, то естественное отображение $\sigma_{\alpha_{J}}: B \rightarrow B_{J}$ определяется следующим образом:

$$
\sigma_{\alpha_{J}}\left(b_{1}, b_{2}, \ldots, b_{m}\right)=\left(b_{j_{1}}, b_{j_{2}}, \ldots, b_{j_{k}}\right) .
$$

Отношение $v\left(\alpha_{\{j\}}\right)$ будем обозначать через $v_{j}$. Очевидно, что $\alpha_{J}=\bigwedge_{j \in J} \alpha_{\{j\}}$. Поэтому

$$
v\left(\alpha_{J}\right)=\underset{j \in J}{\wedge} v_{j}
$$

Таким образом, отображения $v_{j}$ однозначно определяют нижний гомоморфизм v. Покажем, что они определяют и верхний гомоморфизм $\tau$. Для этого положим $\Lambda(\theta)=\left\langle j \mid \theta \leq v_{j}\right\rangle$. Имеем

$$
v\left(\alpha_{\Lambda(\theta)}\right)=\underset{j \in \Lambda(\theta)}{\wedge} v_{j} \geq \theta
$$


откуда следует $\tau(\theta) \leq \alpha_{\Lambda(\theta)}$. С другой стороны, в силу равенства (4.3)

$$
\left(\theta \leq v\left(\alpha_{J}\right)\right) \Leftrightarrow\left(\forall j \in J: \theta \leq v_{j}\right) \Leftrightarrow(J \subseteq \Lambda(\theta)) \Rightarrow\left(\alpha_{\Lambda(\theta)} \leq \alpha_{J}\right),
$$

что дает

$$
(\tau(\theta) \leq \tau(\theta)) \Rightarrow(\theta \leq v(\tau(\theta))) \Rightarrow\left(\alpha_{\Lambda(\theta)} \leq \tau(\theta)\right) .
$$

Таким образом,

$$
\tau(\theta)=\alpha_{\Lambda(\theta)}
$$

Теорема 4.1. Отображение $f: A \rightarrow \prod_{i=1}^{m} B_{i}$ принадлежит множеству $\mathfrak{F}(A, B ; v)$ тогда и только тогда, когда

$$
f=(\underset{i=1}{\mathrm{~m}}) h_{i} \pi_{v_{i}}: A \rightarrow \prod_{i=1}^{m} B_{i}
$$

для произвольных отображений $h_{i}: A / \xi\left(v_{i}\right) \rightarrow B_{i}$.

Как мы уже отмечали, отображение $f$ однозначно представляется в виде $f=(\stackrel{m}{\times}) f_{i}$ для подходящих отображений $f_{i}: A \rightarrow B_{i}$.

Пусть $J=\left\{j_{1}, j_{2}, \ldots, j_{k}\right\}$. Учитывая равенство (4.2), получаем

$$
\sigma_{\alpha_{J}} f(a)=\sigma_{\alpha_{J}}\left(f_{1}(a), f_{2}(a), \ldots, f_{m}(a)\right)=\left(f_{j 1}(a), f_{j 2}(a), \ldots, f_{j k}(a)\right),
$$

откуда следует $\operatorname{ker} \sigma_{\alpha_{J}} f=\bigwedge_{j \in J} \operatorname{ker} f_{j}$. Отсюда, из равенства (4.3) и определений следует, что включение $f \in \mathfrak{F}(A, B ; v)$ имеет место тогда и только тогда, когда для любого подмножества $J$ выполняется включение

$$
\widehat{\jmath}_{j \in J} \operatorname{ker} f_{j} \geq \widehat{\curlywedge}_{j \in J} v_{j} .
$$

В частности, взяв в качестве $J$ одноэлементное множество $\{i\}$, получаем, что в случае $f \in \mathfrak{F}(A, B ; v)$ справедливо включение

$$
\operatorname{ker} f_{i} \geq v_{i} .
$$


Обратно: легко видеть, что если включения (4.7) справедливы для всех номеров $i \in\{1, \ldots, m\}$, то включения (4.6) справедливы для всех подмножеств $J$ и, следовательно, $f \in \mathfrak{F}(A, B ; v)$.

Осталось заметить, что включение (4.7) справедливо тогда и только тогда, когда $f_{i}=h_{i} \pi_{v_{i}}$ для подходящего отображения $h_{i}$.

Отображение $f$, для которого выполняется равенство (4.5), будем обозначать через $f_{h_{1} \ldots h_{m}}$.

Из доказанного утверждения легко следует, что мощность $M$ множества $\mathfrak{F}(A, B ; v)$ равна $M=\prod_{i=1}^{m}\left|B_{i}\right|^{\left|A / \xi\left(v_{i}\right)\right|}$.

Лемма 4.1. Пусть $a_{1}, a_{2} \in A, b_{1} ; b_{2} \in B$. Тогда для того, чтобы сущеествовало такое отображение $f \in \mathfrak{F}(A, B ; v)$, что

$$
f\left(a_{1}\right)=b_{1}, \quad f\left(a_{2}\right)=b_{2},
$$

необходимо и достаточно, чтобы выполнялось неравенство

$$
\tau\left(\llbracket a_{1}, a_{2} \rrbracket\right) \geq \llbracket b_{1}, b_{2} \rrbracket .
$$

Если это неравенство выполняется, то существует ровно $\frac{M}{|B|^{2}}\left|B / \tau\left(\llbracket a_{1}, a_{2} \rrbracket\right)\right|$ функиий $f \in \mathfrak{F}(A, B ; v)$, для которых выполняются равенства (4.8).

Пусть $b_{j}=\left(b_{j 1}, \ldots, b_{j m}\right)$. Тогда $\llbracket b_{1}, b_{2} \rrbracket=\alpha_{J\left(b_{1}, b_{2}\right)}$, где $J_{b_{1} b_{2}}=\left\langle i \mid b_{1 i}=b_{2 i}\right\rangle$. Из равенства (4.4) следует, что неравенство (4.9) эквивалентно включению

$$
\Lambda\left(\llbracket a_{1}, a_{2} \rrbracket\right) \subseteq J_{b_{1} b_{2}} .
$$

Рассмотрим отображение $f_{h_{1} \ldots h_{m}} \in \mathfrak{F}(A, B ; v)$. Ясно, что равенства (4.8) выполняются тогда и только тогда, когда для каждого номера $i \in\{1, \ldots, m\}$ выполняются равенства

$$
h_{i}\left(\pi_{v_{i}}\left(a_{1}\right)\right)=b_{1 i}, \quad h_{i}\left(\pi_{v_{i}}\left(a_{2}\right)\right)=b_{2 i} .
$$

Допустим, что неравенство (4.10) не выполняется. Это означает, что существует такой номер $i$, что $i \in \Lambda\left(\llbracket a_{1}, a_{2} \rrbracket\right), i \notin J_{b_{1} b_{2}}$. По определению, первое включение означает, что $\llbracket a_{1}, a_{2} \rrbracket \leq v_{i}$, откуда следует, что $\left(a_{1}, a_{2}\right) \in \xi\left(v_{i}\right)$ 
и $\pi_{v_{i}}\left(a_{1}\right)=\pi_{v_{i}}\left(a_{2}\right)$. Далее, $b_{1 i} \neq b_{2 i}$ в силу $i \notin J_{b_{1} b_{2}}$. Теперь ясно, что для этого номера равенства (4.11) не могут выполняться. Следовательно, не могут выполняться и равенства (4.8).

Теперь будем считать, что неравенство (4.10) выполнено. Выберем произвольный номер $i \in \Lambda\left(\llbracket a_{1}, a_{2} \rrbracket\right)$. При сделанном предположении $i \in J_{b_{1} b_{2}}$. Опять же из определений следует, что в этом случае выполняются равенства $\pi_{v_{i}}\left(a_{1}\right)=\pi_{v_{i}}\left(a_{2}\right)$ и $b_{1 i}=b_{2 i}$. Очевидно, что в этом случае существует ровно $\left|B_{i}\right|^{\left|A / \xi\left(v_{i}\right)\right|-1}$ функций $h_{i}$, для которых равенства (4.11) выполняются.

В оставшемся случае $i \notin \Lambda\left(\llbracket a_{1}, a_{2} \rrbracket\right)$ выполняется неравенство $\pi_{v_{i}}\left(a_{1}\right) \neq \pi_{v_{i}}\left(a_{2}\right)$. Очевидно, что в этом случае существует ровно $\left.\left.\left|B_{i}\right|\right|^{4 / v_{i}}\right|^{-2}$ функций $h_{i}$, для которых равенства (4.11) выполняются.

Окончательно получаем, что существует ровно

$$
\begin{gathered}
\prod_{i \in \Lambda\left(\llbracket a_{1}, a_{2} \rrbracket\right)}\left|B_{i}\right|^{\left|A / \xi\left(v_{i}\right)\right|-1} \prod_{i \notin \Lambda\left(\llbracket a_{1}, a_{2} \rrbracket\right)}\left|B_{i}\right|^{\left|A / \xi\left(v_{i}\right)\right|-2}= \\
=\frac{\prod_{i \in \Lambda\left(\llbracket a_{1}, a_{2} \rrbracket\right)}\left|B_{i}\right|}{\prod_{i=1}^{m}\left|B_{i}\right|^{2}} \prod_{i=1}^{m}\left|B_{i}\right|^{\left|A / \xi\left(v_{i}\right)\right|}=\frac{M}{|B|^{2}}\left|B / \tau\left(\llbracket a_{1}, a_{2} \rrbracket\right)\right|
\end{gathered}
$$

функций, для которых выполняются равенства (4.8).

Теперь очевидно

Следствие 4.1. Пусть $\varphi-$ случайное отображение, имеющее равномерное распределение на множестве $\mathfrak{F}(A, B ; v)$. Тогда оно является слабо коррелированным.

Таким образом, формула (3.14) и полученные на ее основе теорема 3.2 и следствие 3.1 применимы к случайному уравнению $\Phi$ с независимой правой частью, левая часть которого имеет равномерное распределение на множестве $\mathfrak{F}(A, B ; v)$, и согласованному с ним заведомо разрешимому уравнению $\Psi$.

\section{Список литературы}

1. Балакин, Г. В. Введение в теорию случайных уравнений // В сб.: Труды по дискретной математике. - М.: ТВП, 1997. — Т. 1. - С. 1-18. 
2. Боровков, A. А. Теория вероятностей. - М.: Эдиториал УРСС, 1999.

3. Горчинский, Ю. Н. О гомоморфизмах многоосновных универсальных алгебр в связи с криптографическими приложениями // В сб.: Труды по дискретной математике. - М.: ТВП, 1997. - Т. 1. - С. 67-84.

4. Гретиер, Г. Общая теория решеток. - М.: Мир, 1982.

5. Курош, А. Г. Лекции по общей алгебре. - М.: Наука, 1973.

6. Полин, С. В. Решение уравнений методом последовательного группирования и его оптимизация // Математические вопросы криптографии. 2012. - Т. 3. Вып. 1. - С. 97-123.

7. Сачков, $B . H$. Введение в комбинаторные методы дискретной математики. - М.: Наука, 1982.

8. Ширяев, А. Н. Вероятность-1. - М.: МЦНМО, 2004.

9. Шнайер, Б. Прикладная криптография. Протоколы, алгоритмы, исходные тексты на языке Си. - М.: Триумф, 2002.

10. Иосида, К. Функциональный анализ. - М.: Мир, 1967. 
